\title{
Renormalized holographic entanglement entropy in Lovelock gravity
}

\author{
Giorgos Anastasiou, ${ }^{a}$ Ignacio J. Araya, ${ }^{b}$ Robert B. Mann ${ }^{c}$ and Rodrigo Olea ${ }^{d}$ \\ ${ }^{a}$ Instituto de Física, Pontificia Universidad Católica de Valparaíso, \\ Casilla 4059, Valparaíso, Chile \\ ${ }^{b}$ Instituto de Ciencias Exactas y Naturales, Facultad de Ciencias, Universidad Arturo Prat, \\ Avenida Arturo Prat Chacón 2120, 1110939, Iquique, Chile \\ ${ }^{c}$ Department of Physics and Astronomy, University of Waterloo, \\ Waterloo, Ontario, N2L 3G1, Canada \\ ${ }^{d}$ Departamento de Ciencias Físicas, Universidad Andres Bello, \\ Sazié 2212, Piso 7, Santiago, Chile \\ E-mail: georgios.anastasiou@pucv.cl, ignaraya@unap.cl, \\ rbmann@uwaterloo.ca, rodrigo.olea@unab.cl
}

ABSTRACT: We study the renormalization of Entanglement Entropy in holographic CFTs dual to Lovelock gravity. It is known that the holographic EE in Lovelock gravity is given by the Jacobson-Myers (JM) functional. As usual, due to the divergent Weyl factor in the Fefferman-Graham expansion of the boundary metric for Asymptotically AdS spaces, this entropy functional is infinite. By considering the Kounterterm renormalization procedure, which utilizes extrinsic boundary counterterms in order to renormalize the on-shell Lovelock gravity action for AAdS spacetimes, we propose a new renormalization prescription for the Jacobson-Myers functional. We then explicitly show the cancellation of divergences in the EE up to next-to-leading order in the holographic radial coordinate, for the case of spherical entangling surfaces. Using this new renormalization prescription, we directly find the $C$-function candidates for odd and even dimensional CFTs dual to Lovelock gravity. Our results illustrate the notable improvement that the Kounterterm method affords over other approaches, as it is non-perturbative and does not require that the Lovelock theory has limiting Einstein behavior.

KEYwords: AdS-CFT Correspondence, Classical Theories of Gravity, Gauge-gravity correspondence

ArXiv EPrint: 2103.14640 


\section{Contents}

1 Introduction 1

2 Preliminaries: Lovelock gravity and factorized equations of motion 4

3 Renormalized Lovelock-AdS action $\quad 6$

3.1 The $P(\mathcal{F})$ formulation for even-dimensional AAdS manifolds $\quad 7$

4 Renormalized Lovelock-AdS action on the replica orbifold and HEE 10

5 Renormalized HEE divergence cancellation in Lovelock-AdS 12

6 Topological reinterpretation of HEE in odd-dimensional CFTs 15

7 Renormalized HEE for ball-shaped regions and $C$-function candidates 18

8 Conclusions 20

$\begin{array}{ll}\text { A Generalized Kronecker delta identities } & 22\end{array}$

B Factorization of the Lovelock equation of motion 23

B.1 EGB case 24

$\begin{array}{lll}\text { C } & P(\mathcal{F}) \text { and degeneracy conditions } & 24\end{array}$

$\begin{array}{ll}\text { D Noether prepotential from } P(\mathcal{F}) & 27\end{array}$

D.1 Degenerate case 28

E JM extremal surface for ball-shaped entangling region $\quad 29$

F Evaluation of the JM functional and co-dimension 2 Kounterterm $\quad 30$

\section{Introduction}

Entanglement entropy has played an significant role in advancing our understanding of holography. The Ryu-Takayanagi (RT) prescription [1] allows one to compute the entanglement entropy for a region $A$ in a conformal field theory (CFT) dual to Einstein gravity (and additional matter fields) in terms of the area $\mathcal{A}\left(\Sigma_{A}\right)$ of a surface $\Sigma_{A}$ of minimal area in the bulk that is homologous to $A$

$$
S_{\mathrm{EE}}(A)=\frac{\mathcal{A}\left(\Sigma_{A}\right)}{4 G}
$$


known as the Holographic Entanglement Entropy (HEE) formula, where $G$ is the gravitational constant.

Higher curvature terms likewise enrich our understanding of holography. They appear as quantum (or stringy) corrections to Einstein gravity $[2,3]$ and in general have holographic duals that are not equivalent to those defind from Einstein gravity [4]. They have been used to investigate interesting CFT physics [5-10] that in some cases is quite universal, applicable to very general CFTs [11-20].

Higher-curvature terms generalize both boundary terms of the action functional [21$24]$ and the Bekenstein- Hawking black hole entropy-area relation [25, 26] in terms of the Wald formula [27, 28], and it is natural to expect they will modify the entanglement entropy formula in (1.1). However employing the expected Wald functional fails [29] because the extrinsic curvature(s) of the generalized bulk surface must be taken into account. This was first carried out for quadratic gravity [30], and then a general formula was obtained for theories whose actions have arbitrary contractions of the Riemann tensor [31-34]. However this formula involved taking a weighted sum over trace anomaly charges whose evaluation, beyond quadratic order, entails a theory-dependent splitting of the Riemann tensor components that is somewhat complicated. This shortcoming was recently circumvented [35] in terms of a general formula obtained in terms of implicit derivatives of a Euclidean higher curvature action with respect to projections of the Riemann tensor and extrinsic curvature tensors associated with the RT surface $\Sigma_{A}$.

We obtain in this paper the renormalization of the HEE formula, suitable for any Lovelock theory of gravity [36], which is

$$
S_{E E}^{\mathrm{ren}}(A)=S_{J M}\left[\Sigma_{A}\right]+\frac{c_{d}\left\lfloor\frac{d+1}{2}\right\rfloor}{4 G} \int_{\partial \Sigma} B_{d-2},
$$

where $\Sigma_{A}$ is the codimension-2 surface that extremizes the Jacobson-Myers (JM) functional $S_{J M}[\Sigma][37], c_{d}$ is a dimension-dependent constant and $B_{d-2}$ is an extrinsic boundary counterterm (both given below in eqs. (3.2) and (3.3)) and $\lfloor x\rfloor$ is the usual floor function. We obtain this by making use of the Kounterterm renormalization procedure [38-40]. This procedure has been successfully applied to computations for holographic CFTs dual to Einstein-AdS gravity [41-44]. It was recently used to obtain an expression for the conserved charges of solutions having $k$-fold degenerate vacua in Lovelock AdS gravity, making manifest a link between the degeneracy of a given vacuum and the nonlinearity of the energy formula $[45,46]$.

The prescription (1.2) exploits the replica formula, whose holographic implementation $[47,48]$ generalized the minimal area prescription (1.1) beyond the sphericallysymmetric case [49]. The entanglement entropy in the saddle-point approximation of AdS/CFT is given by

$$
S_{E E}=-\lim _{\alpha \rightarrow 1} \partial_{\alpha} I_{E}\left[M_{d+1}^{(\alpha)}\right],
$$

where $I_{E}\left[M_{d+1}^{(\alpha)}\right]$ is the Euclidean on-shell action for the bulk, evaluated on a suitably constructed $(d+1)$-dimensional conically singular orbifold $M_{d+1}^{(\alpha)}$ (with angular deficit 
given by $2 \pi(1-\alpha))$. In the case of Einstein-AdS [31], $M_{d+1}^{(\alpha)}$ is the backreacted manifold sourced by a codimension- 2 cosmic brane with tension $T=\frac{(1-\alpha)}{4 G}$, coupled to the ambient geometry through the Nambu-Goto (NG) action. In the $\alpha \rightarrow 1$ tensionless limit, the NG action decouples from the ambient geometry and the extremal codimension- 2 surface that represents the on-shell location of the brane becomes the usual RT surface.

The computation of HEE is therefore directly related to the evaluation of on-shell gravity actions, such that the divergences in the former are entirely due to the divergences in the latter. It then becomes evident that if one considers the renormalized on-shell action, the entropies thus computed will be renormalized as well.

In the Kounterterm approach, renormalization of the on-shell Einstein-AdS action is carried out by considering extrinsic boundary counterterms (Kounterterms) [38, 39]. The usual asymptotic charges and thermodynamic behavior of asymptotically AdS (AAdS) black-hole solutions are correctly recovered. Furthermore, the agreement of this extrinsic counterterm renormalization procedure with the standard Holographic Renormalization scheme [50] has been demonstrated for a large class of AAdS spaces [51]. The Kountertermrenormalized action has been evaluated on $M_{d+1}^{(\alpha)}$ orbifolds [43], based on the work of Fursaev, Patrushev and Solodukhin [30]. In doing so, via eq. (1.3), the renormalized HEE was readily computed.

We are interested here in holographic CFTs that are dual to Lovelock gravity. In order to compute the holographic EE in this case, we note that eq. (1.3) is independent of the particular type of dual gravity theory. This is because (1.3) assumes only that the AdS/CFT correspondence holds, namely that the gravitational theory of choice is the correct dual of the CFT under study. This, in turn, implies that the saddle-point approximation is valid, such that the partition function of the CFT is given by the exponential of (minus) the Euclidean on-shell gravity action of the dual bulk manifold. Then, one need only evaluate $I_{E}\left[M_{d+1}^{(\alpha)}\right]$ for the corresponding gravity theory and (1.3) still applies. We thereby obtain eq. (1.2) using logic similar to that in Einstein-AdS gravity, employing the renormalization of the on-shell Lovelock gravity action developed in refs. [40, 52], and applying it to conically singular manifolds [43].

We consider even and odd dimensional CFTs separately. For odd dimensional CFTs, the renormalized HEE in eq. (1.2) can be rewritten in terms of the intrinsic AdS curvature $\mathcal{F}$ of the minimal surface $\Sigma_{A}$ (defined below in eq. (6.5)) and the Euler characteristic of $\Sigma_{A}$. For spherical entangling surfaces only the topological number contributes (as we will show in section 7). In both odd and even dimensional CFTs, the HEE counterterm given by the $B_{d-2}$ term in eq. (1.2) is explicitly shown to cancel the leading order divergence coming from the JM functional. It also cancels the next-to-leading order divergence in the case of spherical entangling surfaces in conformally flat AdS boundaries. The renormalized EE obtained here corresponds to the finite part for odd-dimensional CFTs and the logarithmically divergent part for even-dimensional CFTs. In both cases these quantities are universal, as they are related to the holographic $C$-function candidate of the CFT, which for the odd- $d$ case is the $a^{*}$ charge [53] and for the even- $d$ case is the type- $A$ anomaly coefficient [31, 32]. 
Our paper is organized as follows. In section 2, we review the Lovelock-AdS gravity theory, emphasizing the equation of motion (EOM) and its factorization in terms of the different maximally-symmetric configurations for the vacua of the theory. In section 3 we revisit the renormalized Lovelock-AdS action [40], and in the case of even-dimensional bulk manifolds, we rewrite the action in terms of a polynomial $P(\mathcal{F})$ in the AdS curvature with respect to a chosen vacuum [54]. In section 4 , we evaluate the renormalized Lovelock-AdS action on the replica orbifold, in order to obtain the contribution of the co-dimension 2 extremal surface to the action, and from there, the renormalized HEE. We also review the derivation of the Euler-Lagrange equation for the extremal surface of the JM functional, which defines the co-dimension 2 surface whose JM entropy gives the HEE. We show how to write this equation in a new factorized form. In section 5, we exhibit the explicit cancellation of leading and next-to-leading order divergences in the renormalized HEE. In section 6, we consider odd-dimensional holographic CFTs dual to Lovelock theory and we decompose the HEE into a geometric part written as a polynomial on the AdS curvature $\mathcal{F}$ of the intrinsic metric of the extremal surface $\Sigma$, and a purely topological part that depends on the Euler characteristic of $\Sigma$. In section 7, we consider the example of ballshaped entangling regions in the CFT and we compute the renormalized HEE for both odd-dimensional and even-dimensional CFTs, relating the resulting universal part to the $a^{*}$ charge (or generalized $F$ quantity) and type-A anomaly coefficient respectively, both of which are $C$-function candidates. In section 8 , we summarize our results for the HEE and relate them to the holographic properties of the CFT. We also give some general conclusions based on our results and discuss possible future avenues of research.

\section{Preliminaries: Lovelock gravity and factorized equations of motion}

Lovelock gravity is the most general pure gravity action such that it has second order differential equations for the dynamical variable, i.e., the metric [36]. The Lovelock action is given by

$$
I_{L}\left[M_{d+1}\right]=\frac{1}{16 \pi G} \int_{M_{d+1}} d^{d+1} x \sum_{p=0}^{\left\lfloor\frac{d}{2}\right\rfloor} \alpha_{p} L_{2 p},
$$

where the Lovelock densities $L_{2 p}$ are defined by

$$
L_{2 p}=\frac{1}{2^{p}} \sqrt{-\mathcal{G}} \delta_{\mu_{1} \cdots \mu_{2 p}}^{\nu_{1} \cdots \nu_{2 p}} R_{\nu_{1} \nu_{2}}^{\mu_{1} \mu_{2}} \cdots R_{\nu_{2 p-1} \nu_{2 p}}^{\mu_{2 p-1} \mu_{2 p}}
$$

$\delta_{\mu_{1} \cdots \mu_{2 p}}^{\nu_{1} \cdots \nu_{2 p}}$ is the generalized Kronecker-delta, and $\lfloor x\rfloor$ is the integer floor of $x$. We note that Einstein-AdS gravity is a particular case of the Lovelock action defined in eq. (2.1), for which

$$
\alpha_{0}=-2 \Lambda=\frac{d(d-1)}{\ell^{2}}, \quad \alpha_{1}=1,
$$

and $\alpha_{i}=0$ for $i>1$. The Lovelock theories we shall consider are higher-curvature corrections to Einstein-AdS gravity and therefore, unless otherwise stated, the values of $\alpha_{0}$ and $\alpha_{1}$ are always given as in eq. (2.3). 
The Lovelock EOM is given by $[36,46]$

$$
E_{\mu}^{\nu}=\sum_{p=0}^{\left\lfloor\frac{d}{2}\right\rfloor} \frac{\alpha_{p}}{2^{p+1}} \delta_{\mu \mu_{1} \cdots \mu_{2 p}}^{\nu \nu_{1} \cdots \nu_{2 p}} R_{\nu_{1} \nu_{2}}^{\mu_{1} \mu_{2}} \cdots R_{\nu_{2 p-1} \nu_{2 p}}^{\mu_{2 p-1} \mu_{2 p}}=0,
$$

and using the values of $\alpha_{0}$ and $\alpha_{1}$ given in eq. (2.3), it can be rewritten as

$$
\begin{aligned}
R_{\nu}^{\mu}-\frac{1}{2}(R-2 \Lambda) \delta_{\nu}^{\mu} & =H_{\nu}^{\mu}, \\
H_{\nu}^{\mu} & =\sum_{p=2}^{\left\lfloor\frac{d}{2}\right\rfloor} \frac{\alpha_{p}}{2^{p+1}} \delta_{\nu \mu_{1} \cdots \mu_{2 p}}^{\mu \nu_{1} \cdots \nu_{2 p}} R_{\nu_{1} \nu_{2}}^{\mu_{1} \mu_{2}} \cdots R_{\nu_{2 p-1} \nu_{2 p}}^{\mu_{2 p-1} \mu_{2 p}},
\end{aligned}
$$

where $H_{\nu}^{\mu}$ is the Lanczos-Lovelock tensor.

As usual, by considering the maximally-symmetric (constant curvature) ansatz for the Riemann curvature tensor in AdS, given by

$$
R_{\nu_{1} \nu_{2}}^{\mu_{1} \mu_{2}}=-\frac{1}{\ell_{\mathrm{eff}}^{2}} \delta_{\nu_{1} \nu_{2}}^{\mu_{1} \mu_{2}}
$$

and inserting it into the EOM (2.4), we obtain a condition for $\ell_{\text {eff }}^{-2}$

$$
\Delta\left(\ell_{\text {eff }}^{-2}\right) \stackrel{\text { def. }}{=} \sum_{p=0}^{\left\lfloor\frac{d}{2}\right\rfloor} \frac{(-1)^{p+1}(d-2) ! \alpha_{p}}{(d-2 p) !}\left(\frac{1}{\ell_{\text {eff }}^{2}}\right)^{p}=0,
$$

given in terms of the characteristic polynomial of the theory [46]. Thus, the roots of $\Delta\left(\ell_{\text {eff }}^{-2}\right)=0$ give the possible effective AdS radii for the vacua of the Lovelock theory characterized by the set of $\left\{\alpha_{p}\right\}$ couplings.

It is easy to see that the roots obtained from eq. (2.8) may have algebraic multiplicity higher than one, which in turn implies that the vacua of the corresponding Lovelock theory are degenerate. By simple algebra considerations, the $k$-th degeneracy condition is defined as

$$
\Delta^{(k)}=\frac{1}{k !} \frac{d^{k} \Delta}{d\left(\ell_{\text {eff }}^{-2}\right)^{k}}=\sum_{p=k}^{\left\lfloor\frac{d}{2}\right\rfloor} \frac{(-1)^{p+1}(d-2) ! p ! \alpha_{p}}{k !(p-k) !(d-2 p) !}\left(\frac{1}{\ell_{\text {eff }}^{2}}\right)^{p-k}=0,
$$

in agreement with ref. [46]. A theory is $(k-1)$-degenerate if the largest algebraic multiplicity of its vacua is $k$, which in turn means that all $\Delta^{(q)}$ for $q<k$ are zero. Note that the normalization of the degeneracy conditions as considered in eq. (2.9) is such that the value of $\Delta^{(1)}$ for Einstein-AdS gravity is equal to one.

As shown in appendix B, the EOM (2.4) can be rewritten in factorized form as

$$
\begin{aligned}
E_{\nu}^{\mu} & =\frac{\alpha_{N}}{2^{N+1}} \delta_{\nu \mu_{1} \cdots \mu_{2 N}}^{\mu \nu_{1} \cdots \nu_{2 N}}\left(R_{\nu_{1} \nu_{2}}^{\mu_{1} \mu_{2}}+\frac{1}{\ell_{\mathrm{eff}(1)}^{2}} \delta_{\nu_{1} \nu_{2}}^{\mu_{1} \mu_{2}}\right) \cdots\left(R_{\nu_{2 N-1} \nu_{2 N}}^{\mu_{2 N-1} \mu_{2 N}}+\frac{1}{\ell_{\mathrm{eff}(N)}^{2}} \delta_{\nu_{2 N-1} \nu_{2 N}}^{\mu_{2 N-1} \mu_{2 N}}\right) \\
& =0
\end{aligned}
$$


where $N \leq\left\lfloor\frac{d}{2}\right\rfloor$ is the order (in powers of the Riemann curvature) of the Lovelock Lagrangian and $\left\{\ell_{\operatorname{eff}(i)}\right\}$ are the effective AdS radii of the theory, given by the solutions of the characteristic polynomial of eq. (2.8) [54]. When the theory has $(k-1)$-degenerate vacua, the term corresponding to the $i$-th degenerate vacuum is repeated $k$ times in the product.

Having reviewed the equations of motion for Lovelock gravity theories, we proceed in the next section with their renormalization. Especially in the case of even-dimensional bulks, a useful rewriting of the renormalized action in terms of a polynomial on the AdS curvature of the manifold is obtained, which is a generalization of the renormalized volume formula proposed for Einstein-AdS [43].

\section{Renormalized Lovelock-AdS action}

We consider the renormalized Lovelock-AdS action, given by [40]

$$
I_{L}^{\mathrm{ren}}\left[M_{d+1}\right]=\frac{1}{16 \pi G} \int_{M_{d+1}} \sum_{p=0}^{\left\lfloor\frac{d}{2}\right\rfloor} \alpha_{p} L_{2 p}+\frac{c_{d}}{16 \pi G} \int_{\partial M_{d+1}} d^{d} x B_{d}
$$

where the $L_{2 p}$ Lovelock densities are defined in eq. (2.2), and the coupling $c_{d}$ is defined by

$$
c_{d}=\left\{\begin{array}{ll}
\frac{2}{d+1} \sum_{p=1}^{\frac{d-1}{2}} \frac{p \alpha_{p}(-1)^{\frac{d+3}{2}-p}}{(d+1-2 p) !}\left(\ell_{\mathrm{eff}}\right)^{(d+1-2 p)} & \text { for odd } d \\
\frac{2}{d}\left[\frac{(d-1) !}{2^{d-2}\left[\left(\frac{d}{2}-1\right) !\right]^{2}}\right] \sum_{p=1}^{\frac{d}{2}} \frac{p \alpha_{p}(-1)^{\frac{d}{2}+1-p}}{(d+1-2 p) !}\left(\ell_{\mathrm{eff}}\right)^{(d-2 p)} & \text { for even } d
\end{array} .\right.
$$

Here, $\ell_{\text {eff }}$ is the effective AdS radius of the branch under consideration. Also, the boundary Kounterterm is given by

$$
B_{d}=\left\{\begin{array}{c}
-(d+1) \sqrt{-h} \int_{0}^{1} d t \delta_{i_{1} \cdots i_{d}}^{j_{1} \cdots j_{d}} K_{j_{1}}^{i_{1}}\left(\frac{1}{2} \mathcal{R}_{j_{2} j_{3}}^{i_{2} i_{3}}-t^{2} K_{j_{2}}^{i_{2}} K_{j_{3}}^{i_{3}}\right) \cdots\left(\frac{1}{2} \mathcal{R}_{j_{d-1} j_{d}}^{i_{d-1} i_{d}}-t^{2} K_{j_{d-1}}^{i_{d-1}} K_{j_{d}}^{i_{d}}\right) \\
\text { for odd } d \\
-d \sqrt{-h} \int_{0}^{1} d t \int_{0}^{t} d s \delta_{i_{1} \cdots i_{d-1}}^{j_{1} \cdots j_{d-1}} K_{j_{1}}^{i_{1}}\left(\frac{1}{2} \mathcal{R}_{j_{2} j_{3}}^{i_{2} i_{3}}-t^{2} K_{j_{2}}^{i_{2}} K_{j_{3}}^{i_{3}}+\frac{s^{2}}{\ell_{\mathrm{eff}}^{2}} \delta_{j_{2}}^{i_{2}} \delta_{j_{3}}^{i_{3}}\right) \times \\
\cdots\left(\frac{1}{2} \mathcal{R}_{\left.{ }_{j_{d-2} j_{d-1}}^{i_{d-2} i_{d-1}} t^{2} K_{j_{d-2}}^{i_{d-2}} K_{j_{d-1}}^{i_{d-1}}+\frac{s^{2}}{\ell_{\mathrm{eff}}^{2}} \delta_{j_{d-2}}^{i_{d-2}} \delta_{j_{d-1}}^{i_{d-1}}\right)}^{\text {for even } d},\right.
\end{array},\right.
$$

where $K_{j}^{i}$ is the extrinsic curvature of the foliation with respect to the radial coordinate $\rho$ and $\mathcal{R}^{i_{1} i_{2}}{ }_{j_{1} j_{2}}$ is the Riemann curvature of the intrinsic metric $h$ in the foliation $[40,52]$. We emphasize that $\ell_{\text {eff }}$ in eq. (3.2) is the effective AdS radius of the vacuum (maximally symmetric) solution about which the action is renormalized. In other words, the renormalized action evaluated in that vacuum is zero, and for solutions that are continuously connected to that vacuum (by the value of the corresponding black hole charges) it measures the free energy with respect to said vacuum. The action defined in eq. (3.1) has a finite value as well as a well-defined variational principle for a large class of solutions including black holes with rotation and electromagnetic charges [40]. 


\subsection{The $\boldsymbol{P}(\mathcal{F})$ formulation for even-dimensional AAdS manifolds}

The renormalized volume of an even-dimensional AAdS-Einstein manifold can be defined as a polynomial in totally antisymmetric contractions of the tensor [43]

$$
\mathcal{F}_{\nu_{1} \nu_{2}}^{\mu_{1} \mu_{2}}=R_{\nu_{1} \nu_{2}}^{\mu_{1} \mu_{2}}+\frac{1}{\ell^{2}} \delta_{\nu_{1} \nu_{2}}^{\mu_{1} \mu_{2}}
$$

It can be checked that this definition matches the standard definition of renormalized volume in $D=4$ (for generic Poincaré-Einstein manifolds) and 6 (only for asymptotically conformally flat manifolds) as found in the mathematical literature [55-59]. In this section, we find the analogous $P(\mathcal{F})$ polynomial corresponding to the Kounterterm-renormalized bulk Lovelock action in even-dimensional AAdS manifolds.

The Euler theorem states that for odd $d$

$$
\int_{M} E_{d+1}-\int_{\partial M} d^{d} x B_{d}=(4 \pi)^{\frac{d+1}{2}}\left(\frac{d+1}{2}\right) ! \chi(M),
$$

where $E_{d+1}$ is the Euler density of the $(d+1)$-dimensional bulk, $B_{d}$ is the boundary Chern form defined in eq. (3.3) and $\chi(M)$ is the Euler characteristic of the bulk manifold. In this case we can rewrite the renormalized Lovelock-AdS action (3.1) as

$$
I_{L}^{\mathrm{ren}}[M]=\frac{1}{16 \pi G} \int_{M}\left(\sum_{p=0}^{\frac{d-1}{2}} \alpha_{p} L_{2 p}+c_{d} E_{d+1}\right)+\tau_{d} \chi(M),
$$

where

$$
\tau_{d}=-\frac{c_{d}}{16 \pi G}(4 \pi)^{\frac{d+1}{2}}\left(\frac{d+1}{2}\right) ! .
$$

Upon defining the following quantities as

$$
\alpha_{\frac{d+1}{2}}=c_{d}, \quad L_{d+1}=E_{d+1},
$$

we have that

$$
I_{L}^{\mathrm{ren}}[M]=\frac{1}{16 \pi G} \int_{M}^{\frac{d+1}{2}} \sum_{p=0}^{\frac{1}{2}} \alpha_{p} L_{2 p}+\tau_{d} \chi(M) .
$$

One may rewrite the latter expression using eq. (3.4) such that

$$
\begin{aligned}
I & =16 \pi G\left(I_{L}^{\mathrm{ren}}[M]-\tau_{d} \chi(M)\right) \\
& =\int_{M} d^{d+1} x \sqrt{-\mathcal{G}} \sum_{p=0}^{\frac{d+1}{2}} \frac{\alpha_{p}}{2^{p}} \delta_{\mu_{1} \cdots \mu_{2 p}}^{\nu_{1} \cdots \nu_{2 p}}\left(\mathcal{F}_{\nu_{1} \nu_{2}}^{\mu_{1} \mu_{2}}-\frac{1}{\ell_{\mathrm{eff}}^{2}} \delta_{\nu_{1} \nu_{2}}^{\mu_{1} \mu_{2}}\right) \cdots\left(\mathcal{F}_{\nu_{2 p-1} \nu_{2 p}}^{\mu_{2 p-1} \mu_{2 p}}-\frac{1}{\ell_{\mathrm{eff}}^{2}} \delta_{\nu_{2 p-1} \nu_{2 p}}^{\mu_{2 p-1} \mu_{2 p}}\right) \\
& =\int_{M} d^{d+1} x \sqrt{-\mathcal{G}} \sum_{p=0}^{\frac{d+1}{2}} \sum_{j=0}^{p} \frac{(-1)^{p-j} p !(d+1-2 j) ! \alpha_{p}}{2^{j} j !(p-j) !(d+1-2 p) ! \ell_{\mathrm{eff}}^{2(p-j)}} \delta_{\mu_{1} \cdots \mu_{2 j}}^{\nu_{1} \cdots \nu_{2 j}} \mathcal{F}_{\nu_{1} \nu_{2}}^{\mu_{1} \mu_{2}} \ldots \mathcal{F}_{\nu_{2 j-1} \nu_{2 j}}^{\mu_{2 j-1} \mu_{2 j}},
\end{aligned}
$$

upon using the delta identities given in appendix A. 
We now proceed to show that the coefficients of the $\mathcal{F}^{0}$ and $\mathcal{F}^{1}$ terms are zero. The coefficient of the $\mathcal{F}^{0}$ term is given by

$$
\begin{aligned}
c_{0} & =\sum_{p=0}^{\frac{d+1}{2}} \frac{(-1)^{p}(d+1) ! \alpha_{p}}{(d+1-2 p) !}\left(\frac{1}{\ell_{\text {eff }}^{2}}\right)^{p} \\
& =\sum_{p=0}^{\frac{d-1}{2}} \frac{(-1)^{p}(d+1) ! \alpha_{p}}{(d+1-2 p) !}\left(\frac{1}{\ell_{\text {eff }}^{2}}\right)^{p}-\left(\sum_{p=0}^{\frac{d-1}{2}} \frac{(-1)^{p}(d+1) ! \alpha_{p}}{(d+1-2 p) !}\left(\frac{2 p}{d+1}\right)\left(\frac{1}{\ell_{\text {eff }}^{2}}\right)^{p}\right) \\
& =\sum_{p=0}^{\frac{d-1}{2}} \frac{(-1)^{p} d ! \alpha_{p}}{(d-2 p) !}\left(\frac{1}{\ell_{\text {eff }}^{2}}\right)^{p} \\
& =-d(d-1) \Delta\left(\ell_{\text {eff }}^{-2}\right)=0,
\end{aligned}
$$

using eqs. (3.2) and (3.8). The last line follows from the definition of (any one of) the effective AdS radii given by the characteristic polynomial $\Delta\left(\ell_{\text {eff }}^{-2}\right)=0$ of the Lovelock theory as defined in eq. (2.8), noting that $\left\lfloor\frac{d}{2}\right\rfloor=\left\lfloor\frac{d-1}{2}\right\rfloor$ for odd $d$. Turning to the coefficient of the $\mathcal{F}^{1}$ term, we have

$$
\begin{aligned}
c_{1} & =\sum_{p=1}^{\frac{d+1}{2}} \frac{(-1)^{p-1} p(d-1) ! \alpha_{p}}{2(d+1-2 p) ! \ell_{\mathrm{eff}}^{2(p-1)}} \\
& =\sum_{p=1}^{\frac{d-1}{2}}\left(\frac{(-1)^{p-1} p(d-1) ! \alpha_{p}}{2(d+1-2 p) ! \ell_{\mathrm{eff}}^{2(p-1)}}+\frac{(-1)^{d}(-1)^{p-1} p(d-1) ! \alpha_{p}}{2(d+1-2 p) ! \ell_{\mathrm{eff}}^{2(p-1)}}\right) \\
& =0
\end{aligned}
$$

considering again eqs. (3.2) and (3.8) and noting that $(-1)^{d}=-1$.

Thus to lowest order in the AdS curvature, the integrand $P(\mathcal{F})$ in eq. (3.10) is of quadratic and higher order in $\mathcal{F}$, which (as discussed in appendix B) also implies that the Noether prepotential is proportional to $\mathcal{F}$ at the normalizable order (assuming a nondegenerate theory). We then have that

$$
I_{L}^{\mathrm{ren}}[M]=\frac{1}{16 \pi G} \int_{M} d^{d+1} x \sqrt{-\mathcal{G}} P_{(d+1),\left\{\alpha_{p}\right\}}(\mathcal{F})+\tau_{d} \chi(M),
$$

for the Kounterterm-renormalized Lovelock-AdS action for odd $d$, where

$$
P_{(d+1),\left\{\alpha_{p}\right\}}(\mathcal{F})=\sum_{p=2 j=2}^{\frac{d+1}{2}} \sum^{p} \frac{(-1)^{p-j} p !(d+1-2 j) ! \alpha_{p}}{2^{j} j !(p-j) !(d+1-2 p) ! \ell_{\mathrm{eff}}^{2(p-j)}} \delta_{\mu_{1} \cdots \mu_{2 j}}^{\nu_{1} \cdots \nu_{2 j}} \mathcal{F}_{\nu_{1} \nu_{2}}^{\mu_{1} \mu_{2}} \ldots \mathcal{F}_{\nu_{2 j-1} \nu_{2 j}}^{\mu_{2 j-1} \mu_{2 j}},
$$

and with $\tau_{d}$ given in terms of the Euler characteristic of the bulk manifold $M_{d+1}$ from (3.7). We note that sometimes it is more convenient to write

$$
P_{(d+1),\left\{\alpha_{p}\right\}}(\mathcal{F})=\sum_{j=2}^{\frac{d+1}{2}} c_{j} \delta_{\mu_{1} \cdots \mu_{2 j}}^{\nu_{1} \cdots \nu_{2 j}} \mathcal{F}_{\nu_{1} \nu_{2}}^{\mu_{1} \mu_{2}} \ldots \mathcal{F}_{\nu_{2 j-1} \nu_{2 j}}^{\mu_{2 j-1} \mu_{2 j}}
$$


in order to immediately identify the coefficient $c_{j}$ of the $\mathcal{F}^{j}$ term as

$$
c_{j}=\sum_{p=j}^{\frac{d+1}{2}} \frac{(-1)^{p-j} p !(d+1-2 j) ! \alpha_{p}}{2^{j} j !(p-j) !(d+1-2 p) ! \ell_{\mathrm{eff}}^{2(p-j)}} .
$$

In the Einstein-AdS case, where $\alpha_{0}$ and $\alpha_{1}$ are given in (2.3), with $\alpha_{p>1}=0$, the expression (3.15) matches that obtained previously for the definition of renormalized volume [43].

We show in appendix $\mathrm{C}$ that

$$
c_{k+1}=\sum_{i=1}^{k} p_{(k+1, i)} \Delta^{(i)},
$$

for some coefficients $p_{(k+1, i)}$ (given in eq. (C.8)), in agreement with the previous results for Lovelock gravity with $k$-fold degenerate vacua [45]. We can explicitly write the first three factors as

$$
\begin{aligned}
& c_{2}=\frac{\ell_{\mathrm{eff}}^{2}}{2^{3} 2 !(d-2)}\left(\Delta^{(1)}\right), \\
& c_{3}=-\frac{\ell_{\mathrm{eff}}^{4}}{2^{5} 3 !(d-2)(d-4)}\left(\Delta^{(1)}+\frac{4}{(d-3) \ell_{\mathrm{eff}}^{2}} \Delta^{(2)}\right), \\
& c_{4}=\frac{\ell_{\mathrm{eff}}^{6}}{2^{7} 4 !(d-2)(d-4)(d-6)}\left(\Delta^{(1)}+\frac{4}{(d-3) \ell_{\mathrm{eff}}^{2}} \Delta^{(2)}+\frac{24}{(d-3)(d-5) \ell_{\mathrm{eff}}^{4}} \Delta^{(3)}\right) .
\end{aligned}
$$

Also, the generic factor is given by

$$
\begin{aligned}
c_{i}=\frac{(-1)^{i} \ell_{\mathrm{eff}}^{2 i-2}}{2^{2 i-1} i !(d-2)(d-4) \cdots(d+2-2 i)} & \left(\Delta^{(1)}+\frac{4}{(d-3) \ell_{\mathrm{eff}}^{2}} \Delta^{(2)}\right. \\
& \left.+\frac{24}{(d-3)(d-5) \ell_{\mathrm{eff}}^{4}} \Delta^{(3)}+\cdots+\frac{2^{(i-1)} i !}{\ell_{\mathrm{eff}}^{2(i-2)}(d-3)(d-5) \cdots(d+3-2 i)} \Delta^{(i-1)}\right) .
\end{aligned}
$$

Taking advantage of eq. (3.17) we can write

$$
P_{(d+1),\left\{\alpha_{p}\right\}}(\mathcal{F})=\sum_{j=2}^{\frac{d+1}{2} j-1} \sum_{i=1}\left(p_{(j, i)} \Delta^{(i)}\right) \delta_{\mu_{1} \cdots \mu_{2 j}}^{\nu_{1} \cdots \nu_{2 j}} \mathcal{F}_{\nu_{1} \nu_{2}}^{\mu_{1} \mu_{2}} \cdots \mathcal{F}_{\nu_{2 j-1} \nu_{2 j}}^{\mu_{2 j-1} \mu_{2 j}}
$$

thereby relating $P(\mathcal{F})$ to the degeneracy conditions $(2.9)$.

For a $k$-fold degenerate vacuum, all the degeneracy conditions up to (and including) $\Delta^{(k)}$ are zero as seen from their definition in eq. (2.9). Thus, the lowest order in $\mathcal{F}$ of the $P(\mathcal{F})$ that encodes the renormalized action of a $k$-degenerate theory (normalized with respect to the $k$-degenerate vacuum) is $\mathcal{F}^{k+2}$. Furthermore the $P_{(d+1),\left\{\alpha_{p}\right\}}(\mathcal{F})$ can always be written as

$$
P_{(d+1),\left\{\alpha_{p}\right\}}(\mathcal{F})=c_{k+2} \delta_{\mu_{1} \cdots \mu_{2 k+4}}^{\nu_{1} \cdots \nu_{2 k+3}} \mathcal{F}_{\nu_{1} \nu_{2}}^{\mu_{1} \mu_{2}} \cdots \mathcal{F}_{\nu_{2 k+3} \nu_{2 k+4}}^{\mu_{2 k+3} \mu_{2 k+4}}+\sum_{j=k+3}^{\frac{d+1}{2}} c_{j} \delta_{\mu_{1} \cdots \mu_{2 j}}^{\nu_{1} \cdots \nu_{2 j}} \mathcal{F}_{\nu_{1} \nu_{2}}^{\mu_{1} \mu_{2}} \cdots \mathcal{F}_{\nu_{2 j-1} \nu_{2 j}}^{\mu_{2 j-1} \mu_{2 j}},
$$


where

$$
c_{k+2}=p_{(k+2, k+1)} \Delta^{(k+1)}
$$

is proportional to the $\Delta^{(k+1)}$ degeneracy condition and $p_{(k+2, k+1)}$ is given by

$$
p_{(k+2, k+1)}=\frac{\ell_{\mathrm{eff}}^{2}(-1)^{k-1}(d-1-2 k) !}{2^{k+2}(k+1)(d-2) !},
$$

in a $k$-degenerate theory.

Thus, the rewriting of the coefficients in terms of the degeneracy conditions indicates that the Noether prepotential of a $k$-degenerate theory is of order $\mathcal{F}^{k+1}$ at the normalizable order, in accordance to ref. [45]. For more information, see appendix D.

In the next section, we consider the Kounterterm-renormalized Lovelock-AdS action discussed here, together with the Lewkowycz-Maldacena (LM) procedure [47], in order to compute the renormalized HEE by evaluating the action on the replica orbifold.

\section{Renormalized Lovelock-AdS action on the replica orbifold and HEE}

Our next task is to evaluate the renormalized (Euclidean) Lovelock-AdS action on the conically-singular orbifold $M_{d+1}^{(\alpha)}$. The Lovelock densities evaluated on the replica orbifold decompose into the sum of the regular bulk part and a co-dimension 2 Lovelock density localized at the extremal surface $\Sigma$ which corresponds to the fixed-point set of the replica symmetry $[30,44,60]$. In particular

$$
\int_{M_{d+1}^{(\alpha)}} d^{d+1} x \sqrt{\mathcal{G}} L_{2 p}^{(\alpha)}=\int_{M_{d+1}} d^{d+1} x \sqrt{\mathcal{G}} L_{2 p}^{(r)}+4 \pi p(1-\alpha) \int_{\Sigma} d^{d-1} y \sqrt{\gamma} L_{2 p-2},
$$

where $L_{2 p-2}$ is an intrinsic Lovelock density evaluated on the co-dimension 2 surface $\Sigma$ with induced metric $\gamma$. Therefore, the bulk part of the Lovelock action, when evaluated on the orbifold, decomposes as

$$
\begin{aligned}
I_{L}^{\text {bulk }}\left[M_{d+1}^{(\alpha)}\right] & =\frac{1}{16 \pi G} \sum_{p=0}^{\left\lfloor\frac{d}{2}\right\rfloor} \alpha_{p} \int_{M_{d+1}} d^{d+1} x \sqrt{\mathcal{G}} L_{2 p}^{(r)}+(1-\alpha) S_{J M}[\Sigma] \\
S_{J M}[\Sigma] & =\frac{1}{4 G} \sum_{p=1}^{\left\lfloor\frac{d}{2}\right\rfloor} \alpha_{p} p \int_{\Sigma} d^{d-1} y \sqrt{\gamma} L_{2 p-2},
\end{aligned}
$$

where $S_{J M}[\Sigma]$ is precisely the Jacobson-Myers (JM) functional [29, 61]. Thus, in analogy with the Einstein-AdS case, the action on the orbifold is interpreted as the bulk contribution plus the action of a brane with tension $T=\frac{(1-\alpha)}{4 G}$, but coupled to the bulk geometry through the JM functional, which has the form of a co-dimension 2 Lovelock Lagrangian evaluated on the intrinsic metric of the brane.

To evaluate the boundary Kounterterm on the orbifold, we consider the self-replicating property of the $B_{d}$ (defined in eq. (3.3)), for both the odd and even $d$ cases [44]. In 
particular, one writes

$$
\int_{\partial M_{d+1}^{(\alpha)}} d^{d} x B_{d}^{(\alpha)}=\int_{\partial M_{d+1}^{(\alpha)}} d^{d} x B_{d}^{(r)}+4 \pi\left\lfloor\frac{d+1}{2}\right\rfloor(1-\alpha) \int_{\partial \Sigma} d^{d-2} y B_{d-2} .
$$

Then, the evaluation of the counterterm results in

$$
I_{L}^{B_{d}}\left[M_{d+1}^{(\alpha)}\right]=\frac{c_{d}}{16 \pi G} \int_{\partial M_{d+1}^{(\alpha)}} d^{d} x B_{d}^{(r)}+\frac{c_{d}\left\lfloor\frac{d+1}{2}\right\rfloor}{4 G}(1-\alpha) \int_{\partial \Sigma} d^{d-2} y B_{d-2},
$$

where the coupling $c_{d}$ is given in eq. (3.2). Combining eqs. (4.2) and (4.4), we have

$$
\begin{aligned}
I_{L}^{\mathrm{ren}}\left[M_{d+1}^{(\alpha)}\right]= & \frac{1}{16 \pi G}\left(\sum_{p=0}^{\left\lfloor\frac{d}{2}\right\rfloor} \alpha_{p} \int_{M_{d+1}} d^{d+1} x \sqrt{G} L_{2 p}^{(r)}+c_{d} \int_{\partial M_{d+1}^{(\alpha)}} d^{d} x B_{d}^{(r)}\right) \\
& +(1-\alpha)\left(S_{J M}[\Sigma]+\frac{c_{d}\left\lfloor\frac{d+1}{2}\right\rfloor}{4 G} \int_{\partial \Sigma} d^{d-2 y} B_{d-2}\right),
\end{aligned}
$$

what is the renormalized Lovelock-AdS action evaluated on the replica orbifold.

From this expression, we compute the renormalized HEE using the replica formula of the LM prescription, given in eq. (1.3). Starting from the action on $M_{d+1}^{(\alpha)}$, given by eq. (4.5), we obtain

$$
S_{E E}^{\mathrm{ren}}=-\left.\partial_{\alpha} I_{E}^{\mathrm{ren}}\left[M_{d+1}^{(\alpha)}\right]\right|_{\alpha=1}=S_{J M}[\Sigma]+\frac{c_{d}\left\lfloor\frac{d+1}{2}\right\rfloor}{4 G} \int_{\partial \Sigma} d^{d-2} y B_{d-2} \equiv S_{J M}^{\mathrm{ren}}[\Sigma],
$$

what defines the renormalized JM functional. Note that $S_{J M}[\Sigma]$ is the JM functional evaluated on the extremal surface $\Sigma$ that minimizes it. We then have that the renormalized HEE, which directly corresponds to its universal part, is given by the renormalized JM functional.

The extremal surface minimizing this new functional is not affected by the counterterms, since it's only a boundary term that does not affect the dynamics. In the tensionless limit $(\alpha \rightarrow 1)$, there is no back-reaction of the extremal surface on the bulk geometry, as seen by the fact that the contribution to the action from the surface (given by the $J M$ functional in eq. (4.5)) vanishes. Thus, the extremal surface is found by finding determined by the global minimum of $S_{J M}$ by itself. The resulting Euler-Lagrange equations, given in ref. [62], obtain the form below

$$
E_{J M}=2 \mathcal{K}_{\beta}^{\alpha}\left(\sum_{p=0}^{\left\lfloor\frac{d-2}{2}\right\rfloor} \frac{\alpha_{(p+1)}(p+1)}{2^{p+1}} \delta_{\alpha \alpha_{1} \cdots \alpha_{2 p}}^{\beta \beta_{1} \cdots \beta_{2 p}} \widehat{\mathcal{R}}_{\beta_{1} \beta_{2}}^{\alpha_{1} \alpha_{2}} \cdots \widehat{\mathcal{R}}_{\beta_{2 p-1} \beta_{2 p}}^{\alpha_{2 p-1} \alpha_{2 p}}\right)=0,
$$

where $\mathcal{K}_{\beta}^{\alpha}$ is the extrinsic curvature of the surface with respect to the normal direction that is not along the time coordinate, and $\widehat{\mathcal{R}}^{\alpha_{1} \alpha_{2}}{ }_{\beta_{1} \beta_{2}}$ is the intrinsic Riemann curvature of the surface. 
The form of the EOM in eq. (4.7) is very similar to that in eq. (2.4) for Lovelock gravity, but with a very important difference. The co-dimension 2 Lanczos-Lovelock tensor is contracted with the extrinsic curvature of the minimal surface, which comes from the variation of the induced metric. Following the same factorization procedure discussed in appendix B, the eq. (4.7) can be rewritten as

$$
\begin{aligned}
E_{J M}= & \mathcal{K}_{\beta}^{\alpha} \frac{\alpha_{N} N}{2^{N-1}} \delta_{\alpha \alpha_{1} \cdots \alpha_{2 N-2}}^{\beta \beta_{1} \cdots \beta_{2 N-2}}\left(\widehat{\mathcal{R}}_{\beta_{1} \beta_{2}}^{\alpha_{1} \alpha_{2}}+\lambda_{(1)} \delta_{\beta_{1} \beta_{2}}^{\alpha_{1} \alpha_{2}}\right)\left(\widehat{\mathcal{R}}_{\beta_{3} \beta_{4}}^{\alpha_{3} \alpha_{4}}+\lambda_{(2)} \delta_{\beta_{3} \beta_{4}}^{\alpha_{3} \alpha_{3}}\right) \\
& \cdots\left(\widehat{\mathcal{R}}^{\left.\alpha_{2 N-3} \alpha_{2 N-2}{ }_{\beta_{2 N-3} \beta_{2 N-2}}+\lambda_{(N-1)} \delta_{\beta_{2 N-3} \beta_{2 N-2}}^{\alpha_{2 N-3} \alpha_{2 N-2}}\right)=0,}\right.
\end{aligned}
$$

where $N \leq\left\lfloor\frac{d}{2}\right\rfloor$ and $\lambda_{(i)}$ are the roots of the polynomial

$$
D(\lambda) \stackrel{\text { def }}{=} \sum_{p=0}^{N-1} \frac{(-1)^{p}(d-2) ! \alpha_{(p+1)}(p+1)}{(d-2 p-2) !} \lambda^{p}=0 .
$$

Note that the $\lambda_{(i)}$ solutions are different from the roots of $\Delta\left(\ell_{\text {eff }}^{-2}\right)$, which define the vacua of the gravity theory.

Finally, it is easy to check that for a ball-shaped entangling region in a pure AdS bulk (dual to the ground state of a CFT in Minkowski spacetime), the extremal surface of the JM functional is the same as the RT minimal surface (i.e., a spherical hemisphere). We show this in detail in appendix E.

Having obtained the renormalized HEE functional for Lovelock-AdS gravity, we proceed in the next section to show the explicit cancellation of leading order and next-toleading order divergencies of said renormalized HEE.

\section{Renormalized HEE divergence cancellation in Lovelock-AdS}

In what follows, we check the cancellation of divergences in the renormalized HEE $S_{E E}^{\text {ren }}$ given in eq. (4.6), for both even and odd dimensional manifolds, up to the next-to-leading

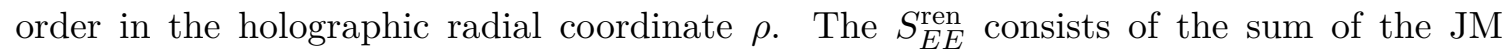
functional (4.2) and the co-dimension 2 Kounterterm, both evaluated on the extremal surface $\Sigma$. The induced metric $\gamma_{\alpha \beta}$ of this extremal surface $\Sigma$ has an FG-like expansion

$$
\begin{aligned}
d s_{\gamma}^{2} & =\gamma_{\alpha \beta} d y^{\alpha} d y^{\beta}=N^{2}(\rho) d \rho^{2}+\widetilde{\gamma}_{a b} d y^{a} d y^{b}, & & \\
N^{2}(\rho) & =\frac{\ell^{2}}{4 \rho^{2}}\left(1+\frac{\rho \ell^{2} \kappa_{a}^{\hat{\imath} a} \kappa_{b}^{\hat{\imath} b}}{(d-2)^{2}}+\ldots\right), & \widetilde{\gamma}_{a b} & =\frac{\sigma_{a b}}{\rho}, \\
\sigma_{a b} & =\sigma_{a b}^{(0)}+\rho \sigma_{a b}^{(2)}+\ldots, & \sigma_{a b}^{(2)} & =-\ell^{2} S_{a b}-\frac{\ell^{2}}{(d-2)} \kappa_{c}^{\hat{\imath} c} \kappa_{a b}^{\hat{\imath}},
\end{aligned}
$$

where $\kappa_{a b}^{\hat{\imath}}$ is the extrinsic curvature of the boundary of the extremal surface $\partial \Sigma$ along the $\hat{\imath}$ direction (normal to the radial coordinate $\rho$ ) and $\widetilde{\gamma}_{a b}$ is the induced metric on $\partial \Sigma[44$, 63, 64]. Furthermore, $\widetilde{\gamma}_{a b}$ has an FG-like expansion whose leading and next-to-leading order coefficients are given by $\sigma^{(0)}$ and $\sigma^{(2)}$, where $\sigma^{(2)}$ depends on the Schouten tensor $S_{a b}$ of the CFT metric $g_{i j}^{(0)}$ evaluated with the indices on $\partial \Sigma$. Note that the Greek letters 
denote directions along the world-volume of $\Sigma$, whereas the lower case Latin letters denote directions along $\partial \Sigma$.

Decomposing the Riemann curvature tensor along and orthogonal to the holographic radial foliation, we obtain

$$
\begin{aligned}
& \widehat{\mathcal{R}}^{\rho a}{ }_{\rho b}=\frac{1}{N} \partial_{\rho} k_{b}^{a}-k_{c}^{a} k_{b}^{c}, \\
& \widehat{\mathcal{R}}^{a \rho}{ }_{b c}=\frac{2}{N} \nabla_{[b} k_{c]}^{a} \quad \widehat{\mathcal{R}}^{a b}{ }_{c \rho}=2 N \nabla^{[a} k_{c}^{b]}, \\
& \widehat{\mathcal{R}}^{a b}{ }_{c d}=\mathcal{R}^{a b}{ }_{c d}-2 k_{c}^{[a} k_{d}^{b]},
\end{aligned}
$$

using the Gauss-Codazzi relations [65], where $k_{b}^{a}$ is the extrinsic curvature of $\partial \Sigma$ along the radial direction $\partial_{\rho}$, and $\nabla_{a}$ is the covariant derivative with respect to $\widetilde{\gamma}_{a b}$. In order to avoid confusion, we denote the Riemann tensor of $\gamma_{\alpha \beta}$ with a hat $\left(\widehat{\mathcal{R}}_{\beta_{1} \beta_{2}}^{\alpha_{1} \alpha_{2}}\right)$, and that of $\widetilde{\gamma}_{a b}$ without a hat $\left(\mathcal{R}_{b_{1} b_{2}}^{a_{1} a_{2}}\right)$. Finally, the FG-like expansion of the co-dimension 3 curvatures (Riemannian curvature and extrinsic curvature along $\partial_{\rho}$ ) at the boundary $\partial \Sigma$ is given by

$$
\begin{aligned}
\mathcal{R}_{b_{1} b_{2}}^{a_{1} a_{2}} & =\rho \mathcal{R}_{b_{1} b_{2}}^{a_{1} a_{2}}[\sigma]=\rho\left(\mathcal{R}^{(0)}\right)^{a_{1} a_{2}}{ }_{b_{1} b_{2}}+\ldots, k_{b}^{a}=\left(k^{(0)}\right)_{b}^{a}+\rho\left(k^{(2)}\right)_{b}^{a}+\ldots, \\
\left(k^{(0)}\right)_{b}^{a} & =\frac{1}{\ell_{\mathrm{eff}}} \delta_{b}^{a}, \quad\left(k^{(2)}\right)_{b}^{a}=-\frac{1}{\ell_{\mathrm{eff}}}\left[\left(\sigma^{(2)}\right)_{b}^{a}+\frac{\left.\ell_{\mathrm{eff}}^{2} \kappa_{c}^{\hat{\imath} c} \kappa_{d}^{\hat{\imath} d} \delta_{b}^{a}\right],}{2(d-2)},\right.
\end{aligned}
$$

where we note that the (0)-quantities are computed with respect to the intrinsic metric $\sigma_{a b}^{(0)}$ on the entangling surface in the CFT [44]. Although the FG-like expansions in eqs. (5.1) and (5.3) were used in ref. [44], they are also valid for Lovelock-AdS.

We now define

$$
\begin{aligned}
4 G S_{E E}^{\mathrm{ren}} & =I_{J M}+I_{K T}, \\
I_{J M} & =\int_{\Sigma} d^{d-1} y \sqrt{\gamma} \sum_{p=0}^{\left\lfloor\frac{d-2}{2}\right\rfloor} \alpha_{(p+1)}(p+1) L_{2 p}, \quad I_{K T}=c_{d}\left\lfloor\frac{d+1}{2}\right\rfloor \int_{\partial \Sigma} d^{d-2} y B_{d-2}, \\
L_{2 p} & =\frac{1}{2^{p}} \delta_{\alpha_{1} \cdots \alpha_{2 p}}^{\beta_{1} \cdots \beta_{2 p}} \widehat{\mathcal{R}}_{\beta_{1} \beta_{2}}^{\alpha_{1} \alpha_{2}} \cdots \widehat{\mathcal{R}}_{\beta_{2 p-1} \beta_{2 p}}^{\alpha_{2 p-1} \alpha_{2 p}}
\end{aligned}
$$

with $c_{d}$ given in eq. (3.2), and proceed with the computation of $I_{J M}$ and $I_{K T}$.

In order to isolate the divergences coming from the bulk term at $\Sigma$, we first expand the $I_{J M}$ of eq. (5.4) in the radial foliation. Using the antisymmetry of the generalized Kronecker delta, $I_{J M}$ can be expanded as

$$
\begin{aligned}
& I_{J M}=I_{J M}^{(1)}+I_{J M}^{(2)}+I_{J M}^{(3)} \\
& I_{J M}^{(1)}=\int_{\Sigma} d^{d-1} y \sqrt{\gamma} \sum_{p=1}^{\left\lfloor\frac{d-2}{2}\right\rfloor} \frac{(p+1) p \alpha_{(p+1)}}{2^{p-2}} \delta_{b_{1} \ldots b_{2 p-1}}^{a_{1} \ldots a_{2 p-1}} \widehat{\mathcal{R}}^{\rho b_{1}}{ }_{\rho a_{1}} \widehat{\mathcal{R}}^{b_{2} b_{3}}{ }_{a_{2} a_{3}} \ldots \widehat{\mathcal{R}}^{b_{2 p-2} b_{2 p-1}}{ }_{a_{2 p-2} a_{2 p-1}}, \\
& I_{J M}^{(2)}=\int_{\Sigma} d^{d-1} y \sqrt{\gamma} \sum_{p=2}^{\left\lfloor\frac{d-2}{2}\right\rfloor} \frac{(p+1)(p-1) p \alpha_{(p+1)}}{2^{p-2}} \delta_{b_{1} \ldots b_{2 p-1}}^{a_{1} \ldots a_{2 p-1}} \widehat{\mathcal{R}}^{\rho b_{1}}{ }_{a_{1} a_{2}} \widehat{\mathcal{R}}_{\rho a_{3}}^{b_{2} b_{3}} \widehat{\mathcal{R}}^{b_{4} b_{5}}{ }_{a_{4} a_{5}} \cdots \mathcal{R}^{b_{2 p-2} b_{2 p-1}}{ }_{a_{2 p-2} a_{2 p-1}}, \\
& I_{J M}^{(3)}=\int_{\Sigma} d^{d-1} y \sqrt{\gamma} \sum_{p=0}^{\left\lfloor\frac{d-2}{2}\right\rfloor} \frac{(p+1) \alpha_{(p+1)}}{2^{p}} \delta_{b_{1} \ldots b_{2 p}}^{a_{1} \ldots a_{2 p} p} \widehat{\mathcal{R}}_{a_{1} a_{2} b_{1} b_{2}} \cdots \widehat{\mathcal{R}}_{a_{2 p-1} a_{2 p}}^{b_{2 p-1} b_{2 p}}
\end{aligned}
$$


where we have separated the indices of all the possible terms into those corresponding to coordinates along the worldvolume of $\partial \Sigma$ (denoted by the Latin lowercase letters $a, b$ ) and the radial coordinate $\rho$. Each of these terms can be simplified using eqs. (5.1)-(5.3); as shown in appendix $\mathrm{F}$, the result is

$$
\begin{aligned}
I_{J M}= & \int_{\Sigma} d^{d-1} y \sqrt{\gamma} \sum_{p=0}^{\left\lfloor\frac{d-2}{2}\right\rfloor} \frac{(-1)^{p}(p+1)(d-1) ! \alpha_{(p+1)}}{\ell_{\mathrm{eff}}^{2 p}(d-1-2 p) !} \\
& \times\left(1-\frac{\rho p}{(d-2)(d-1)}\left[2(d-3)\left(\operatorname{tr}\left[\sigma^{(2)}\right]+\frac{\ell_{\mathrm{eff}}^{2} \kappa_{a}^{(i) a} \kappa_{b}^{(i) b}}{2(d-2)}\right)+\ell_{\mathrm{eff}}^{2} \mathcal{R}[\sigma]\right]\right)+\text { h.o. . }
\end{aligned}
$$

Employing the FG-like expansion

$$
\sqrt{\gamma}=\frac{\ell_{\mathrm{eff}} \sqrt{\sigma^{(0)}}}{2 \rho^{\frac{d}{2}}}\left(1+\frac{\rho}{2}\left(\operatorname{tr}\left[\sigma^{(2)}\right]+\frac{\ell_{\mathrm{eff}}^{2} \kappa_{a}^{(i) a} \kappa_{b}^{(i) b}}{(d-2)^{2}}\right)+\mathcal{O}\left(\rho^{2}\right)\right),
$$

of $\sqrt{\gamma}$ and decomposing the volume element on $\Sigma$ into its radial and transverse components, we obtain

$$
\begin{aligned}
& I_{J M}= C_{1}+\int_{\partial \Sigma} \frac{d^{d-2} y \ell \sqrt{\sigma^{(0)}}}{(d-2) \epsilon^{\frac{d-2}{2}}} \sum_{p=0}^{\left\lfloor\frac{d-2}{2}\right\rfloor} \frac{(-1)^{p}(p+1)(d-1) ! \alpha_{(p+1)}}{(d-1-2 p) ! !_{\mathrm{eff}}^{2 p}} \\
& \times\left[1+\frac{\epsilon}{(d-1)(d-4)}\left(-p \ell^{2} \mathcal{R}^{(0)}+\frac{[(d-3)(d-4 p)+2]}{2} \operatorname{tr}\left[\sigma^{(2)}\right]\right.\right. \\
&\left.\left.+\frac{[d-1-2 p(d-3)]}{2(d-2)} \ell^{2} \kappa_{a}^{(\hat{\imath}) a} \kappa_{b}^{(\hat{\imath}) b}\right)\right]+\cdots,
\end{aligned}
$$

after performing the radial integration up to the cutoff scale $\rho=\epsilon$. In this expression, $C_{1}$ is the constant part, which for odd $d$ is universal but for even $d$ depends on the choice of the cutoff $\epsilon$.

Consider next the Kounterterm contribution $I_{K T}$ in eq. (5.4). We show in appendix F that

$$
\begin{aligned}
I_{K T}= & -\left(\frac{d+1}{2}\right)(d-1) c_{d} \int_{\partial \Sigma} d^{d-2} y \sqrt{\widetilde{\gamma}} P I \\
= & -\int_{\partial \Sigma} d^{d-2} y \frac{\sqrt{\sigma^{(0)}}}{(d-2) \epsilon^{\frac{d-2}{2}}} \sum_{p=0}^{\frac{d-3}{2}} \frac{(-1)^{p}(p+1)(d-1) ! \alpha_{(p+1)}}{(d-1-2 p) ! \ell_{\mathrm{eff}}^{2 p-1}} \\
& \times\left(1-\epsilon\left[\frac{\ell_{\mathrm{eff}}^{2}}{2(d-4)} \mathcal{R}^{(0)}+\frac{1}{2} \operatorname{tr}\left(\sigma^{(2)}\right)+\frac{\ell_{\mathrm{eff}}^{2}}{2(d-2)} \kappa_{a}^{(i) a} \kappa_{b}^{(i) b}\right]\right)+\cdots,
\end{aligned}
$$

in both even and odd $d$.

Finally we compute $S_{E E}^{\text {ren }}$ using the expressions for $I_{J M}$ and $I_{K T}$ in eqs. (5.8) and (5.9). It is evident that the leading $O\left(\epsilon^{-\frac{d-2}{2}}\right)$ divergences from $I_{J M}$ and $I_{K T}$ cancel each other 
in full generality. After some algebra, we obtain

$$
\begin{aligned}
S_{E E}^{\mathrm{ren}} & =\frac{I_{J M}+I_{K T}}{4 G} \\
& =\frac{C_{1}}{4 G}+\left(\sum_{p=0}^{\left\lfloor\frac{d-2}{2}\right\rfloor} \frac{(-1)^{p}(p+1)(d-2) ! \alpha_{(p+1)}}{(d-2-2 p) ! \ell_{\mathrm{eff}}^{2 p}}\right) S_{\mathrm{diff}}+\cdots,
\end{aligned}
$$

where

$$
S_{\mathrm{diff}}=\frac{1}{4 G} \int_{\partial \Sigma} \frac{d^{d-2} y \ell_{\mathrm{eff}} \sqrt{\sigma^{(0)}}}{(d-2)(d-4) \epsilon^{\frac{d-4}{2}}}\left(\frac{1}{2} \ell_{\mathrm{eff}}^{2} \mathcal{R}^{(0)}+(d-3) \operatorname{tr}\left[\sigma^{(2)}\right]+\frac{(d-3) \ell_{\mathrm{eff}}^{2}}{2(d-2)} \kappa_{a}^{(\hat{\imath}) a} \kappa_{b}^{(\hat{\imath}) b}\right),
$$

which can be rewritten as

$$
S_{\mathrm{diff}}=\frac{1}{4 G} \int_{\partial \Sigma} \frac{d^{d-2} y \ell_{\mathrm{eff}}^{3} \sqrt{\sigma^{(0)}}}{(d-2)(d-4) \epsilon^{\frac{d-4}{2}}}\left[\delta_{a}^{c} \delta_{b}^{d}\left(W^{(0)}\right)^{a b}{ }_{c d}-\left(\kappa_{d}^{(\hat{\imath}) a} \kappa_{a}^{(\hat{\imath}) d}-\frac{\kappa_{d}^{(\hat{\imath}) d} \kappa_{a}^{(\hat{\imath}) a}}{(d-2)}\right)\right],
$$

where $\left(W^{(0)}\right)_{c d}^{a b}$ is the Weyl tensor computed from the metric $\sigma_{a b}^{(0)}$ [44]. $S_{\text {diff vanishes for }}$ AAdS manifolds with conformally flat boundaries and for ball-shaped entangling regions, thus explicitly verifying the cancellation of divergences up to next-to-leading order in this case.

\section{Topological reinterpretation of HEE in odd-dimensional CFTs}

Having obtained the renormalized HEE for CFTs dual to Lovelock-AdS gravity in arbitrary dimension, we now focus on the odd- $d$ case. In this case, we present a reinterpretation of the $S_{E E}^{\text {ren }}$ in terms of the sum of a topological term, proportional to the Euler characteristic of $\Sigma$, and a piece that is given by a polynomial of the AdS curvature of $\Sigma$, analogous to the bulk $P(\mathcal{F})$ in eq. (3.15).

We start by considering $S_{E E}^{\text {ren }}$ and $S_{J M}[\Sigma]$ as given in eqs. (4.6) and (4.2),respectively. Using the Euler theorem (3.5), we have

$$
\int_{\Sigma} E_{d-1}-\int_{\partial \Sigma} d^{d-2} y B_{d-2}=(4 \pi)^{\frac{d-1}{2}}\left(\frac{d-1}{2}\right) ! \chi(\Sigma),
$$

relating the Euler density $E_{d-1}$ of the extremal surface $\Sigma$ and the Chern form $B_{d-2}$ at its boundary. The topological number $\widehat{\tau}_{d}$ is defined as

$$
\widehat{\tau}_{d}=-\frac{1}{4 G} \frac{(d+1)}{2} c_{d}(4 \pi)^{\frac{d-1}{2}}\left(\frac{d-1}{2}\right) !,
$$

yielding

$$
S \stackrel{\text { def. }}{=} 4 G\left(S_{E E}^{\text {ren }}-\widehat{\tau}_{d} \chi(\Sigma)\right)=\int_{\Sigma} \sum_{p=0}^{\frac{d-1}{2}} \alpha_{(p+1)}(p+1) L_{2 p}[\gamma]
$$


upon exchanging $B_{d-2}$ in favor of $E_{d-1}$ in eq. (4.6), and where we have renamed

$$
\alpha_{\frac{d+1}{2}}=c_{d}=\frac{2}{d+1} \sum_{p=1}^{\frac{d-1}{2}} \frac{p \alpha_{p}(-1)^{\frac{d+3}{2}-p}}{(d+1-2 p) !}\left(\ell_{\mathrm{eff}}\right)^{(d+1-2 p)}, \quad L_{d-1}[\gamma]=E_{d-1} .
$$

Recalling the eq. (5.4) and noting that

$$
\widehat{\mathcal{R}}_{\beta_{1} \beta_{2}}^{\alpha_{1} \alpha_{2}}=\mathcal{F}_{\beta_{1} \beta_{2}}^{\alpha_{1} \alpha_{2}}-\frac{1}{\ell_{\mathrm{eff}}^{2}} \delta_{\beta_{1} \beta_{2}}^{\alpha_{1} \alpha_{2}},
$$

relates the Riemann tensor $\widehat{\mathcal{R}}_{\beta_{1} \beta_{2}}^{\alpha_{1} \alpha_{2}}$ to the AdS curvature $\mathcal{F}_{\beta_{1} \beta_{2}}^{\alpha_{1} \alpha_{2}}$ on $\Sigma$, we can write

$$
\begin{aligned}
& \int_{\Sigma} d^{d-1} y \sqrt{\gamma} P_{(d-1)}(\mathcal{F}) \\
& =\int_{\Sigma} d^{d-1} y \sqrt{\gamma} \sum_{p=0}^{\frac{d-1}{2}} \frac{\alpha_{(p+1)}(p+1)}{2^{p}} \delta_{\beta_{1} \cdots \beta_{2 p}}^{\alpha_{1} \cdots \alpha_{2 p}}\left(\mathcal{F}_{\alpha_{1} \alpha_{2}}^{\beta_{1} \beta_{2}}-\frac{2}{\ell_{\mathrm{eff}}^{2}} \delta_{\alpha_{1}}^{\beta_{1}} \delta_{\alpha_{2}}^{\beta_{2}}\right) \cdots\left(\mathcal{F}_{\alpha_{2 p-1} \alpha_{2 p}}^{\beta_{2 p-1} \beta_{2 p}} \frac{2}{\ell_{\mathrm{eff}}^{2}} \delta_{\alpha_{2 p-1}}^{\beta_{2 p-1}} \delta_{\alpha_{2 p}}^{\beta_{2 p}}\right) \\
& =\int_{\Sigma} d^{d-1} y \sqrt{\gamma} \sum_{p=0 j=0}^{\frac{d-1}{2}} \sum^{p} \frac{p !(d-1-2 j) !(-1)^{p-j}(p+1) \alpha_{(p+1)}}{j !(p-j) ! 2^{j}(d-1-2 p) ! \ell_{\mathrm{eff}}^{2(p-j)}} \delta_{\beta_{1} \cdots \beta_{2 j}}^{\alpha_{1} \cdots \alpha_{2 j}} \mathcal{F}_{\alpha_{1} \alpha_{2}}^{\beta_{1} \beta_{2} \ldots \mathcal{F}_{\alpha_{2 j-1} \alpha_{2 j}}^{\beta_{2 j-1} \beta_{2 j}}} \\
& =\int_{\Sigma} d^{d-1} y \sqrt{\gamma} \sum_{j=0}^{\frac{d-1}{2}} \widehat{c}_{j} \delta_{\beta_{1} \cdots \beta_{2 j}}^{\alpha_{1} \cdots \alpha_{2 j}} \mathcal{F}_{\alpha_{1} \alpha_{2}}^{\beta_{1} \beta_{2} \ldots \mathcal{F}_{\alpha_{2 j-1} \alpha_{2 j}}^{\beta_{2 j-1} \beta_{2 j}},}
\end{aligned}
$$

where the simplification proceeds in a manner analogous to that of the bulk $P(\mathcal{F})$ in section 3.1, and where

$$
\widehat{c}_{j}=\sum_{p=j}^{\frac{d-1}{2}} \frac{p !(d-1-2 j) !(-1)^{p-j}(p+1) \alpha_{(p+1)}}{j !(p-j) ! 2^{j}(d-1-2 p) ! !_{\mathrm{eff}}^{2(p-j)}},
$$

is the coefficient of the $\mathcal{F}^{j}$ term.

Note that the co-dimension $2 \widehat{c}_{j}$ coefficients of $S$ can be directly related to the bulk coefficients $c_{j}$ of the bulk $P(\mathcal{F})$ given in eq. (3.16). In particular, we have

$$
\widehat{c}_{j}=2(j+1) c_{j+1},
$$

and therefore

$$
\int_{\Sigma} d^{d-1} y \sqrt{\gamma} P_{(d-1)}(\mathcal{F})=\int_{\Sigma} d^{d-1} y \sqrt{\gamma} \sum_{j=1}^{\frac{d-1}{2}} 2(j+1) c_{j+1} \delta_{\beta_{1} \cdots \beta_{2 j}}^{\alpha_{1} \cdots \alpha_{2 j}} \mathcal{F}_{\alpha_{1} \alpha_{2}}^{\beta_{1} \beta_{2}} \cdots \mathcal{F}_{\alpha_{2 j-1} \alpha_{2 j}}^{\beta_{2 j-1} \beta_{2 j}}
$$

since $\widehat{c}_{0}=2 c_{1}=0$, as shown in (3.12). Hence we finally obtain

$$
S_{E E}^{\text {ren }}=\frac{1}{4 G} \int_{\Sigma} d^{d-1} y \sqrt{\gamma} P_{(d-1)}(\mathcal{F})+\widehat{\tau}_{d} \chi(\Sigma)
$$

for the renormalized HEE. 
The reinterpretation of $S_{E E}^{\mathrm{ren}}$ given in eq. (6.10) extends the results previously obtained for Einstein-AdS, in refs. [41, 42], to the entire Lovelock class. Namely, the rewriting of the universal part of the HEE in terms of a topological invariant and a curvature-dependent term is a general feature.

Also, the co-dimension $2 P(\mathcal{F})$ obtained in eq. (6.9) has some noteworthy properties. For instance, comparing this object with the corresponding one coming from the bulk as given in eq. (3.14), we notice that $P(\mathcal{F})$ has the expected self-replicating property when going to co-dimension 2, in analogy with the Einstein-AdS case [43]. However, one not only has to replace $d$ by $(d-2)$ but also $\alpha_{p}$ by $(p+1) \alpha_{p+1}$, which comes from the fact that the JM functional has the form of a derivarive with respect to the Riemann curvature of the Lovelock Lagrangian, but evaluated intrinsically on the extremal surface.

For the degenerate cases it is trivial to relate the $\widehat{c}_{j}$ to the degeneracy conditions using eq. (6.8). In particular, from eqs. (C.1) and (C.2) in appendix C, we have

$$
\begin{aligned}
& \widehat{c}_{1}=4 c_{2}=\frac{\ell_{\text {eff }}^{2}}{2^{2}(d-2)} \Delta^{(1)}\left(\ell_{\text {eff }}^{-2}\right), \\
& \widehat{c}_{2}=6 c_{3}=-\frac{\ell_{\text {eff }}^{2}}{(d-2)(d-4) 2^{3}}\left(\frac{1}{(d-3)} \Delta^{(2)}+\frac{\ell_{\text {eff }}^{2}}{4} \Delta^{(1)}\right),
\end{aligned}
$$

and in the general case

$$
\widehat{c}_{j}=2(j+1) c_{j+1}=2(j+1) \sum_{i=1}^{j} p_{(j+1, i)} \Delta^{(i)},
$$

from eq. (3.17) for some coefficients $p_{(j+1, i)}$ (given in eq. (C.8)). In a $k$-fold degenerate theory, the lowest order in $\mathcal{F}$ of the co-dimension 2 polynomial $P_{(d-1)}(\mathcal{F})$ will be $\mathcal{F}^{k+1}$, in an analogous manner to the Noether prepotential discussed in appendix D. Therefore we can write

$$
P_{(d-1)}(\mathcal{F})=\sum_{j=1}^{\frac{d-1}{2}} \sum_{i=1}^{j} 2(j+1)\left(p_{(j+1, i)} \Delta^{(i)}\right) \delta_{\beta_{1} \cdots \beta_{2 j}}^{\alpha_{1} \cdots \alpha_{2 j}} \mathcal{F}_{\alpha_{1} \alpha_{2}}^{\beta_{1} \beta_{2}} \cdots \mathcal{F}_{\alpha_{2 j-1} \alpha_{2 j}}^{\beta_{2 j-1} \beta_{2 j}},
$$

in terms of the degeneracy conditions $\Delta^{(k)}$.

Note that in a $k$-degenerate theory, the co-dimension 2 polynomial $P_{(d-1)}(\mathcal{F})$ can be written as

$$
P_{(d-1)}(\mathcal{F})=\widehat{c}_{k+1} \delta_{\beta_{1} \cdots \beta_{2 k+2}}^{\alpha_{1} \cdots \alpha_{2 k+2}} \mathcal{F}_{\alpha_{1} \alpha_{2}}^{\beta_{1} \beta_{2}} \ldots \mathcal{F}_{\alpha_{2 k+1} \alpha_{2 k+2}}^{\beta_{2 k+1} \beta_{2 k+2}}+\sum_{j=k+2}^{\frac{d-1}{2}} \widehat{c}_{j} \delta_{\beta_{1} \cdots \beta_{2 j}}^{\alpha_{1} \cdots \alpha_{2 j}} \mathcal{F}_{\alpha_{1} \alpha_{2}}^{\beta_{1} \beta_{2}} \ldots \mathcal{F}_{\alpha_{2 j-1} \alpha_{2 j}}^{\beta_{2 j-1} \beta_{2 j}},
$$

where

$$
\widehat{c}_{k+1}=2(k+2) p_{(k+2, k+1)} \Delta^{(k+1)},
$$

for $p_{(k+2, k+1)}$ as defined in eq. (3.23).

We close this section by commenting that $S_{E E}^{\text {ren }}$ for degenerate theories exhibits an interesting robustness property under shape deformations of the entangling region. What 
occurs is that, as for the Einstein-AdS case (in $d=3$ ) [66], the topological part of the renormalized HEE is unchanged under such deformations, whereas the $P_{(d-1)}(\mathcal{F})$ part changes as $\varepsilon^{2}$ to lowest order in the deformation parameter $\varepsilon$. This is a result of the fact $\mathcal{F}$ is second order in $\varepsilon$ and that $P_{(d-1)}(\mathcal{F})$ is of order $\mathcal{F}^{1}$. However, for a $k$-fold degenerate Lovelock theory, $P_{(d-1)}(\mathcal{F})$ is of order $\mathcal{F}^{k+1}$ and higher, and therefore $P_{(d-1)}(\mathcal{F})$ changes as $\varepsilon^{2 k+2}$ to the leading order in $\varepsilon$.

This means that the renormalized EE of a CFT dual to a degenerate theory is robust under shape deformations, and increasingly so the higher its degeneracy. It is interesting to conjecture that this robustness of $S_{E E}^{\text {ren }}$ under such deformations for degenerate Lovelock theories could constrain higher-order correlators in the dual CFT. However, the study of shape deformations falls outside the scope of this work and will be pursued in a future paper.

\section{Renormalized HEE for ball-shaped regions and $C$-function candidates}

We now proceed to compute the renormalized HEE for ball-shaped entangling surfaces. This case is important as the universal part of the HEE, which is equal to the renormalized value, is directly related to the $C$-function candidate of the CFT [44]. The extremal surface $\Sigma$ of the JM functional in this geometry is the hemisphere in the bulk, as discussed in appendix E. We analyze the odd- $d$ and even- $d$ cases separately, as the expressions for $S_{E E}^{\text {ren }}$ are different.

We start with odd-dimensional CFTs. In this case $\Sigma$ (as defined in eq. (E.2) in appendix E) is a constant-curvature surface, topologically equivalent to a ball. It therefore has vanishing AdS curvature $\mathcal{F}_{\text {AdS }}$ and Euler characteristic $\chi(\Sigma)=1$. Using eq. (6.10) we directly obtain

$$
\begin{aligned}
S_{E E}^{\mathrm{ren}} & =\widehat{\tau}_{d} \\
& =\sum_{p=1}^{\frac{d-1}{2}} \frac{(-1)^{\frac{d+1}{2}-p}(4 \pi)^{\frac{d-1}{2}}\left(\frac{d-1}{2}\right) ! p \alpha_{p}}{4 G_{N}(d+1-2 p) !}\left(\ell_{\mathrm{eff}}\right)^{(d+1-2 p)} \\
& =(-1)^{\frac{d-1}{2}}\left(\sum_{p=0}^{\frac{d-3}{2}} \frac{(p+1) \alpha_{p+1}(d-1) !(-1)^{p}}{(d-1-2 p) ! \ell_{\mathrm{eff}}^{2 p}}\right)\left(\frac{(4 \pi)^{\frac{d-1}{2}}\left(\frac{d-1}{2}\right) !}{4 G_{N}(d-1) !} \ell_{\mathrm{eff}}^{d-1}\right),
\end{aligned}
$$

using the definitions of $\widehat{\tau}_{d}$ and $c_{d}$ given in eqs. (6.2) and (3.2). This factorized form is in accord with previous results for the Einstein-AdS case [41, 42]. Indeed, for Einstein gravity, the first factor in the last expression is identically equal to one. This first prefactor corresponds to the usual constant encountered in the linearization of Lovelock gravity.

Note that the result of eq. (7.1) implies that the coefficient $\widehat{\tau}_{d}$ of the topological term in eq. (6.10) can be written as

$$
\widehat{\tau}_{d}=(-1)^{\frac{d-1}{2}} F_{L}=(-1)^{\frac{d-1}{2}} Q_{L} F_{E H},
$$

where

$$
F_{E H}=\left(\frac{(4 \pi)^{\frac{d-1}{2}}\left(\frac{d-1}{2}\right) !}{4 G_{N}(d-1) !} \ell_{\mathrm{eff}}^{d-1}\right), \quad Q_{L}=\left(\sum_{p=0}^{\left\lfloor\frac{d-2}{2}\right\rfloor} \frac{(p+1) \alpha_{p+1}(d-1) !(-1)^{p}}{(d-1-2 p) ! \ell_{\mathrm{eff}}^{2 p}}\right)
$$


so that $F_{E H}$ is the usual generalized $F$-quantity ( $C$-function candidate) for odd dimensional CFTs dual to Einstein-AdS gravity. The quantity $Q_{L}$ is the prefactor that depends on the Lovelock couplings. Thus, it is natural to conjecture that the $C$-function candidate for odd- $d$ CFTs dual to Lovelock-AdS gravity is given directly by $F_{L}$.

For even- $d$ CFTs, dual to odd- $D$ Lovelock-AdS gravity, the universal part of the HEE corresponds to the coefficient of the logarithmically divergent part, which is proportional to the type-A anomaly coefficient of the CFT. To compute the universal part we start from the expression (4.6) for $S_{E E}^{\text {ren }}$, and note that for a spherical hemisphere in the bulk (the $\Sigma$ of eq. (E.2)) its induced metric (E.4) satisfies

$$
\widehat{\mathcal{R}}_{\beta_{1} \beta_{2}}^{\alpha_{1} \alpha_{2}}=-\frac{1}{\ell_{\text {eff }}^{2}} \delta_{\beta_{1} \beta_{2}}^{\alpha_{1} \alpha_{2}},
$$

since it is a constant curvature manifold. Using the definition of the $L_{2 p}$ Lovelock densities in eq. (2.2) and the delta identities of appendix A, we obtain

$$
L_{2 p}[\gamma]=\frac{1}{2^{p}}\left(-\frac{2}{\ell_{\mathrm{eff}}^{2}}\right)^{p} \delta_{\beta_{1} \cdots \beta_{2 p}}^{\alpha_{1} \cdots \alpha_{2 p}} \delta_{\alpha_{1}}^{\beta_{1}} \cdots \delta_{\alpha_{2 p}}^{\beta_{2 p}} \sqrt{\gamma}=\frac{(d-1) !}{(d-1-2 p) !} \frac{(-1)^{p}}{\ell_{\mathrm{eff}}^{2 p}} \sqrt{\gamma}
$$

Therefore, the JM functional becomes

$$
\begin{aligned}
S_{J M} & =\frac{1}{4 G} \int_{\Sigma} \sqrt{\gamma} d^{d-1} y \sum_{p=0}^{(d-2) / 2} \alpha_{(p+1)}(p+1) \frac{(d-1) !}{(d-1-2 p) !} \frac{(-1)^{p}}{\ell_{\mathrm{eff}}^{2 p}} \\
& =\left(\sum_{p=0}^{(d-2) / 2} \alpha_{(p+1)}(p+1) \frac{(d-1) !}{(d-1-2 p) !} \frac{(-1)^{p}}{\ell_{\mathrm{eff}}^{2 p}}\right) \frac{\operatorname{Area}(\Sigma)}{4 G} .
\end{aligned}
$$

On the other hand, the contribution to the entropy from the Kounterterms is given by

$$
S_{E E}^{\mathrm{KT}}=\frac{d}{2} \frac{c_{d}}{4 G} \int_{\partial \Sigma} d^{d-2} y B_{d-2},
$$

where $c_{d}$ is defined in eq. (3.2). The universal part of the HEE is thus given by

$$
\begin{aligned}
S_{E E}^{\mathrm{Univ}}= & S_{J M}+S_{E E}^{\mathrm{KT}} \\
= & \left(\left(\sum_{p=0}^{(d-2) / 2} \alpha_{(p+1)}(p+1) \frac{(d-1) !}{(d-1-2 p) !} \frac{(-1)^{p}}{\ell_{\mathrm{eff}}^{2 p}}\right) \frac{\operatorname{Area}(\Sigma)}{4 G}\right. \\
& \left.+\frac{1}{4 G}\left[\frac{(d-1) !}{2^{d-2}\left[\left(\frac{d}{2}-1\right) !\right]^{2}}\right]_{p=0}^{\frac{d-2}{2}} \frac{(p+1) \alpha_{p+1}(-1)^{\frac{d}{2}-p}}{(d-1-2 p) !}\left(\ell_{\mathrm{eff}}\right)^{(d-2 p-2)} \int_{\partial \Sigma} B_{d-2}\right) \\
= & \frac{1}{4 G}\left(\sum_{p=0}^{\frac{d-2}{2}} \frac{(p+1) \alpha_{p+1}(d-1) !(-1)^{p}}{(d-1-2 p) ! \ell_{\mathrm{eff}}^{2 p}}\right)\left(\operatorname{Area}(\Sigma)-\frac{d}{2}\left[\frac{(-1)^{\frac{d}{2}}\left(\ell_{\mathrm{eff}}\right)^{(d-2)}}{2^{d-3} d\left[\left(\frac{d}{2}-1\right) !\right]^{2}} \int_{\partial \Sigma} B_{d-2}\right)\right. \\
= & \left(\sum_{p=0}^{\frac{d-2}{2}} \frac{(p+1) \alpha_{p+1}(d-1) !(-1)^{p}}{(d-1-2 p) ! \ell_{\mathrm{eff}}^{2 p}}\right) \frac{\operatorname{Area} \operatorname{Univ}(\Sigma)}{4 G}
\end{aligned}
$$


where

$$
\operatorname{Area}_{\text {Univ }}(\Sigma) \stackrel{\text { def. }}{=} \text { Area }(\Sigma)-\frac{d}{2}\left[\frac{(-1)^{\frac{d}{2}}\left(\ell_{\text {eff }}\right)^{(d-2)}}{2^{d-3} d\left[\left(\frac{d}{2}-1\right) !\right]^{2}}\right] \int_{\partial \Sigma} d^{d-2} y B_{d-2}
$$

is independent of any Lovelock factors.

In the Einstein-AdS case the universal part of the HEE is precisely equal to $\frac{\operatorname{Area}_{\mathrm{Univ}}(\Sigma)}{4 G}$, such that Area Univ exactly matches the universal (logarithmically divergent) part of the area for the minimal (RT) surface [44]. In other words

$$
\frac{\text { Area }_{\text {Univ }}(\Sigma)}{4 G}=(-1)^{\frac{d}{2}+1} 4\left[\frac{\ell_{\mathrm{eff}}^{d-1} \pi^{\frac{d}{2}-1}}{8 G\left(\frac{d}{2}-1\right) !}\right] \ln \left(\frac{L}{\delta}\right)
$$

where $\delta$ is a cutoff scale in the Poincaré coordinate $z \stackrel{\text { def. }}{=} \ell \sqrt{\rho}$, which scales as a length and $L$ is the radius of the entangling region in the CFT. We see from eq. (7.8) that this quantity likewise governs the universal part of the HEE in Lovelock gravity, so that

$$
S_{E E}^{\mathrm{Univ}}=(-1)^{\frac{d}{2}+1} 4\left(\sum_{p=0}^{\frac{d-2}{2}} \frac{(p+1) \alpha_{p+1}(d-1) !(-1)^{p}}{(d-1-2 p) ! \ell_{\mathrm{eff}}^{2 p}}\right)\left[\frac{\ell_{\mathrm{eff}}^{d-1} \pi^{\frac{d}{2}-1}}{8 G\left(\frac{d}{2}-1\right) !}\right] \ln \left(\frac{L}{\delta}\right),
$$

for ball-shaped entangling regions in even- $d$ CFTs. We recognize that the factor with the sum is again the usual factor $Q_{L}$ (as given in eq. (7.3)) that appears in solutions to Lovelock gravity. This is identically equal to one in the case of Einstein-AdS gravity, recovering previous results in the Einstein-AdS case [44].

Note that eq. (7.11) can be rewritten as

$$
S_{E E}^{\mathrm{Univ}}=(-1)^{\frac{d}{2}+1} 4 A_{L} \ln \left(\frac{L}{\delta}\right),
$$

such that

$$
A_{L}=Q_{L} A_{E H}, \quad A_{E H}=\frac{\ell_{\mathrm{eff}}^{d-1} \pi^{\frac{d}{2}-1}}{8 G\left(\frac{d}{2}-1\right) !},
$$

with $A_{E H}$ being the type-A anomaly coefficient ( $C$ - function candidate) of even- $d$ CFTs dual to Einstein-AdS. Therefore, it is natural to conjecture that for even- $d$ CFTs dual to Lovelock-AdS, the type-A anomaly coefficient is given directly by $A_{L} \cdot{ }^{1}$

\section{Conclusions}

We have shown how the renormalization of HEE for CFTs dual to Lovelock gravity can be carried out using the Kounterterm procedure. The computation takes advantage of the selfreplicating property of Lovelock densities $L_{2 p}$ [60] and the one of the extrinsic counterterms $B_{d}[44]$, when evaluated on squashed cones. In particular, when evaluated on a cone, both

\footnotetext{
${ }^{1}$ The $C$-function candidate for generic $\mathcal{L}$ (Riemann) theories has been previously written, in ref. [15], as proportional to the Lagrangian evaluated on the AdS vacuum with effective radius $\ell_{\text {eff. }}$ It can be shown that our expression matches said results.
} 
$L_{2 p}$ and the $B_{d}$ split into a regular contribution and the corresponding co-dimension 2 term localized at the conical singularity. This allows us to cast the renormalized HEE into the form (4.6), in agreement with the result of ref. [29] for the bulk term of the JM entropy functional. Furthermore, our result shows that the co-dimension 2 Kounterterm added at the boundary $\partial \Sigma$ of the extremal surface is the structure that renormalizes the entanglement entropy.

In studying the renormalization of the gravity theory, we were able to write the Lovelock Lagrangian $\mathcal{L}$ for even-dimensional bulk manifolds as a polynomial of the AdS curvature $\mathcal{F}$ (3.14). This form is convenient as the coefficients of the different powers of $\mathcal{F}$ are linear combinations of the degeneracy conditions (3.19), extending the analysis of ref. [45] for the Noether prepotential used in the computation of asymptotic charges. Indeed, writing the Lagrangian in terms of the degeneracy conditions makes it clear that any variation of $\mathcal{L}$ and, therefore, the Noether prepotential, will inherit the same property. Furthermore, the relation between the degeneracy conditions and the coefficients of powers of $\mathcal{F}$ is also valid for the renormalized JM functional for odd-dimensional CFTs dual to Lovelock gravity.

It is evident from (6.10) that the finite entanglement entropy contains a topological contribution proportional to the Euler characteristic of the extremal surface. The proportionality constant corresponds to the generalized F-quantity of the theory. Furthermore, a purely geometric term arises at finite order, which can be expressed as a polynomial in $\mathcal{F}$. This extends the known relation between the finite part of the HEE for spherical entangling regions in Einstein gravity and the F-quantity to different shapes and higher Lovelock densities.

For generic Lovelock gravity dual to CFTs of both even and odd dimensions, we have shown the finiteness of our renormalized HEE functional (5.10). Our analysis applies to Lovelock densities of arbitrary degree in the curvature, and the cancellation of the leadingorder term was shown in full generality. The cancellation of the next-to-leading order divergence was verified in the case of manifolds with conformally flat boundaries and for spherical entangling regions.

Our results demonstrate that the Kounterterm scheme efficiently isolates the universal part of the HEE, for both even and odd-dimensional cases. This universal part, for spherical entangling regions, corresponds to the C-function candidate. For odd-dimensional CFTs, this candidate is the F-quantity (proportional to the CFT partition function evaluated on a sphere), whereas for even-dimensional CFTs it is the type-A anomaly coefficient. We obtained explicit formulas for both quantities in eqs. (7.3) and (7.13), respectively, where we note that the C-function candidate is always proportional to the one for Einstein-AdS gravity, with a coupling-dependent factor, commensurate with the recent literature [35, 67].

We emphasize that Kounterterm method constitutes a notable improvement over other approaches as it is non-perturbative, neither assuming that the Lovelock couplings are small, nor that the Lovelock theory has an Einstein behavior. An example of the latter is given by degenerate Lovelock theories, where the method is still applicable for isolating the universal part of the HEE. It is interesting to note (as mentioned in eq. (6.14)) that the resulting renormalized JM functional for odd-dimensional CFTs is more robust under deformations of the entangling region in the degenerate cases. The reason for this is that 
the leading power (in $\mathcal{F}$ ) of the polynomial form of the JM functional depends on the degeneracy condition; for a $k$-degenerate theory it is $\mathcal{F}^{k+1}$. Since the topological part is not affected by continuous deformations of the entangling surface, the resulting effect will enter only through the change in $\mathcal{F}$, which to leading order is quadratic in the deformation parameter. It would be interesting to explore the consequences of this feature, but an analysis of shape deformations falls outside the scope of the present paper.

\section{Acknowledgments}

We thank Alberto Güijosa for interesting discussions and feedback. The work of GA and RO was funded in part by FONDECYT grants No. 3190314 Holographic Complexity from Anti-de Sitter gravity and No. 1170765 Boundary dynamics in anti-de Sitter gravity and gauge/gravity duality. The work of IJA is funded by ANID, REC Convocatoria Nacional Subvención a Instalación en la Academia Convocatoria Año 2020, Folio PAI77200097. The work of RBM was supported in part by the Natural Sciences and Engineering Research Council of Canada.

\section{A Generalized Kronecker delta identities}

In the main text, we use three identities involving the generalized Kronecker delta. The most important one is

$$
\delta_{\nu_{1} \cdots \nu_{k}}^{\mu_{1} \cdots \mu_{k}} \delta_{\mu_{k-p+1}}^{\nu_{k-p+1}} \cdots \delta_{\mu_{k}}^{\nu_{k}}=\frac{(r-k+p) !}{(r-k) !} \delta_{\nu_{1} \cdots \nu_{k-p}}^{\mu_{1} \cdots \mu_{k-p}}
$$

which allows to lower its rank by contracting it with a sequence of rank-1 Kronecker deltas. In the expression above, $r$ is the range of the indices, $k$ is the rank of the generalized Kronecker delta, and $p$ is the number of rank-1 deltas present in the sequence.

The second identity is

$$
\delta_{\nu_{1} \cdots \nu_{k}}^{\mu_{1} \cdots \mu_{k}} \delta_{\mu_{1} \mu_{2}}^{\nu_{1} \nu_{2}} \cdots \delta_{\mu_{2 q-1} \mu_{2 q}}^{\nu_{2 q-1} \nu_{2 q}}=2^{q} \delta_{\nu_{1} \cdots \nu_{k}}^{\mu_{1} \cdots \mu_{k}} \delta_{\mu_{1}}^{\nu_{1}} \cdots \delta_{\mu_{2 q}}^{\nu_{2 q}}
$$

which is simply a consequence of the antisymmetry of the rank-2 delta and of the overall contaction implemented by the rank- $k$ delta.

The third identity follows directly from the previous two identities. It states that

$$
\begin{aligned}
N & =\left\lfloor\frac{d}{2}\right\rfloor, \quad r=d+1, \\
\delta_{\nu \nu_{1} \cdots \nu_{2 p}}^{\mu \mu_{1} \cdots \mu_{2 p}} & =\frac{1}{2^{N-p}(d-2 p) !} \delta_{\nu \nu_{1} \cdots \nu_{2 N}}^{\mu \mu_{1} \cdots \mu_{2 N}} \delta_{\mu_{2 p+1} \mu_{2 p+2}}^{\nu_{2 p+1} \nu_{2 p+2}} \cdots \delta_{\mu_{2 N-1} \mu_{2 N}}^{\nu_{2 N-1} \nu_{2 N}} .
\end{aligned}
$$

In the previous expression, $\lfloor x\rfloor$ is the integer floor of $x$ and $d+1$ is the range of the indices of the deltas. The proof of this identity has to be done separately for odd and even $d$, but it is straightforward. 


\section{B Factorization of the Lovelock equation of motion}

We begin by noting that the Lovelock EOM can be written as

$$
E_{\mu}^{\nu}=\sum_{p=0}^{N} \frac{\alpha_{p}}{2^{p+1}} \frac{1}{2^{N-p}(d-2 p) !} \delta_{\mu \mu_{1} \cdots \mu_{2 N}}^{\nu \nu_{1} \cdots \nu_{2 N}} \delta_{\nu_{2 p+1} \nu_{2 p+2}}^{\mu_{2 p+1} \mu_{2 p+2}} \cdots \delta_{\nu_{2 N-1} \nu_{2 N}}^{\mu_{2 N-1} \mu_{2 N}} R_{\nu_{1} \nu_{2}}^{\mu_{1} \mu_{2}} \cdots R_{\nu_{2 p-1} \nu_{2 p}}^{\mu_{2 p-1} \mu_{2 p}}=0 .
$$

We have rewritten $\delta_{\mu \mu_{1} \cdots \mu_{2 p}}^{\nu \nu_{1} \cdots \nu_{2 p}}$ of eq. (2.4) using the delta identity of eq. (A.3). We now define

$$
A_{\nu_{1} \cdots \nu_{2 N}}^{\mu_{1} \cdots \mu_{2 N}}=\sum_{p=0}^{N} \frac{\alpha_{p} d !}{(d-2 p) !} R_{\nu_{1} \nu_{2}}^{\mu_{1} \mu_{2}} \cdots R_{\nu_{2 p-1} \nu_{2 p}}^{\mu_{2 p-1} \delta_{\nu_{2 p+1} \nu_{2 p+2}}} \cdots \delta_{\nu_{2 N-1} \nu_{2 N}}^{\mu_{2 N-1} \mu_{2 N}}
$$

and thus, the EOM can be rewritten as

$$
\delta_{\mu \mu_{1} \cdots \mu_{2 N}}^{\nu \nu_{1} \cdots \nu_{2 N}} \frac{1}{d ! 2^{N+1}} A_{\nu_{1} \cdots \nu_{2 N}}^{\mu_{1} \cdots \mu_{2 N}}=0 .
$$

The tensor $A$ can then be factorized in a straightforward manner

$$
A_{\nu_{1} \cdots \nu_{2 N}}^{\mu_{1} \cdots \mu_{2 N}}=\sum_{p=0}^{N} \frac{\alpha_{p} d !}{(d-2 p) !} x_{\nu_{1} \nu_{2}}^{\mu_{1} \mu_{2}} \cdots x_{\nu_{2 p-1} \nu_{2 p}}^{\mu_{2 p-1} \mu_{2 p}} y_{\nu_{2 p+1} \nu_{2 p+2}}^{\mu_{2 p+1} \mu_{2 p+2}} \cdots y_{\nu_{2 N-1} \nu_{2 N}}^{\mu_{2 N-1} \mu_{2 N}}
$$

by writing

$$
x_{\nu_{1} \nu_{2}}^{\mu_{1} \mu_{2}} \stackrel{\text { def. }}{=} R_{\nu_{1} \nu_{2}}^{\mu_{1} \mu_{2}}, \quad y_{\nu_{1} \nu_{2}}^{\mu_{1} \mu_{2}} \stackrel{\text { def. }}{=} \delta_{\nu_{1} \nu_{2}}^{\mu_{1} \mu_{2}}
$$

Then, $A$, as a polynomial, can be decomposed into its roots as

$$
\begin{aligned}
A_{\nu_{1} \cdots \nu_{2 N}}^{\mu_{1} \cdots \mu_{2 N}} & =\alpha_{N} d !\left(x_{\nu_{1} \nu_{2}}^{\mu_{1} \mu_{2}}-\left(x_{(1)}\right)_{\nu_{1} \nu_{2}}^{\mu_{1} \mu_{2}}\right) \cdots\left(x_{\nu_{2 N-1} \nu_{2 N}}^{\mu_{2 N-1} \mu_{2 N}}-\left(x_{(N)}\right)_{\nu_{2 N-1} \nu_{2 N}}^{\mu_{2 N-1} \mu_{2 N}}\right), \\
\left(x_{(i)}\right)_{\nu_{2 i-1} \nu_{2 i}}^{\mu_{2 i-1} \mu_{2 i}} & =-\lambda_{(i)} y_{\nu_{2 i-1} \nu_{2 i}}^{\mu_{2 i-1} \mu_{2 i}}
\end{aligned}
$$

where $\left\{\lambda_{(i)}\right\}$ are the solutions of

$$
\sum_{p=0}^{N} \frac{\alpha_{p} d !}{(d-2 p) !}(-1)^{p} \lambda^{p}=0
$$

As a consequence, the equations of motion (B.1) can be cast as

$$
E_{\mu}^{\nu}=\delta_{\mu \mu_{1} \cdots \mu_{2 N}}^{\nu \nu_{1} \cdots \nu_{2 N}} \frac{\alpha_{N}}{2^{N+1}}\left(R_{\nu_{1} \nu_{2}}^{\mu_{1} \mu_{2}}+\lambda_{(1)} \delta_{\nu_{1} \nu_{2}}^{\mu_{1} \mu_{2}}\right) \cdots\left(R_{\nu_{2 N-1} \nu_{2 N}}^{\mu_{2 N-1} \mu_{2 N}}+\lambda_{(N)} \delta_{\nu_{2 N-1} \nu_{2 N}}^{\mu_{2 N-1} \mu_{2 N}}\right)=0
$$

where $\lambda_{(i)}$ is solution of

$$
\sum_{p=0}^{N} \frac{\alpha_{p} d !}{(d-2 p) !}(-1)^{p} \lambda^{p}=-d(d-1) \Delta(\lambda)=0
$$

and the polynomial $\Delta(\lambda)$ is defined in eq. (2.8). 
Hence, the EOM (B.7) in factorized form is

$$
E_{\mu}^{\nu}=\frac{\alpha_{N}}{2^{N+1}} \delta_{\mu \mu_{1} \cdots \mu_{2 N}}^{\nu \nu_{1} \cdots \nu_{2 N}}\left(R_{\nu_{1} \nu_{2}}^{\mu_{1} \mu_{2}}+\frac{1}{\ell_{\mathrm{eff}(1)}^{2}} \delta_{\nu_{1} \nu_{2}}^{\mu_{1} \mu_{2}}\right) \cdots\left(R_{\nu_{2 N-1} \nu_{2 N}}^{\mu_{2 N-1} \mu_{2 N}}+\frac{1}{\ell_{\mathrm{eff}(N)}^{2}} \delta_{\nu_{2 N-1} \nu_{2 N}}^{\mu_{2 N-1} \mu_{2 N}}\right)=0,
$$

where the $\ell_{\text {eff }(i)}^{2}$ are the effective AdS radii corresponding to the vacua of the theory. If the $i$-th vacuum is $\left(k_{i}-1\right)$ - degenerate, the corresponding factor will appear $k_{i}$ terms in the factorization such that $k_{i}$ is its algebraic multiplicity, and the sum of all algebraic multiplicities is equal to $N$.

\section{B.1 EGB case}

In the particular case of EGB theory, $\alpha_{0}$ and $\alpha_{1}$ are given in eq. (2.3), and of the higher curvature couplings, only $\alpha_{2} \neq 0$. Thus, we have

$$
\begin{aligned}
\Delta(\lambda) & =\sum_{p=0}^{N} \frac{\alpha_{p}(d-2) !}{(d-2 p) !}(-1)^{p+1} \lambda^{p} \\
& =-\frac{1}{\ell^{2}}+\lambda-(d-2)(d-3) \alpha_{2} \lambda^{2}=0,
\end{aligned}
$$

yielding

$$
\lambda=\frac{-1 \pm \sqrt{1-\frac{4(d-2)(d-3) \alpha_{2}}{\ell^{2}}}}{-2(d-2)(d-3) \alpha_{2}}=\frac{1 \mp \sqrt{1-\frac{4(d-2)(d-3) \alpha_{2}}{\ell^{2}}}}{2(d-2)(d-3) \alpha_{2}},
$$

from which we have

$$
\ell_{\mathrm{eff}(\mp)}^{2}=\frac{2(d-2)(d-3) \alpha_{2}}{1 \mp \sqrt{1-\frac{4(d-2)(d-3) \alpha_{2}}{\ell^{2}}}} .
$$

Thus, the EOM factorizes as

$$
E_{\nu}^{\mu}=\frac{\alpha_{2}}{2^{3}} \delta_{\nu \mu_{1} \mu_{2} \mu_{3} \mu_{4}}^{\mu \nu_{1} \nu_{2} \nu_{3} \nu_{4}}\left(R_{\nu_{1} \nu_{2}}^{\mu_{1} \mu_{2}}+\frac{1}{\ell_{\mathrm{eff}(-)}^{2}} \delta_{\nu_{1} \nu_{2}}^{\mu_{1} \mu_{1}}\right)\left(R_{\nu_{3} \nu_{4}}^{\mu_{3} \mu_{4}}+\frac{1}{\ell_{\mathrm{eff}(+)}^{2}} \delta_{\nu_{3} \nu_{4}}^{\mu_{3} \mu_{4}}\right) .
$$

The degenerate case corresponds to

$$
\alpha_{2}=\frac{\ell^{2}}{4(d-2)(d-3)}, \quad \ell_{\mathrm{eff}(\mp)}^{2}=\frac{\ell^{2}}{2} \Rightarrow \Delta^{(1)}=0
$$

and so

$$
E_{\nu}^{\mu}=\frac{\alpha_{2}}{2^{3}} \delta_{\nu \mu_{1} \mu_{2} \mu_{3} \mu_{4}}^{\mu \nu_{1} \nu_{2} \nu_{3} \nu_{4}}\left(R_{\nu_{1} \nu_{2}}^{\mu_{1} \mu_{2}}+\frac{2}{\ell^{2}} \delta_{\nu_{1} \nu_{2}}^{\mu_{1} \mu_{1}}\right)\left(R_{\nu_{3} \nu_{4}}^{\mu_{3} \mu_{4}}+\frac{2}{\ell^{2}} \delta_{\nu_{3} \nu_{4}}^{\mu_{3} \mu_{4}}\right)=0 .
$$

\section{C $\quad P(\mathcal{F})$ and degeneracy conditions}

We now study how the degeneracy conditions introduced in eq. (2.9) appear in the coefficients of $P(\mathcal{F})$. Recalling (3.2) and (3.8), from eq. (3.15), the $\mathcal{F}^{2}$ coefficient is given 
by

$$
\begin{aligned}
c_{2}= & \sum_{p=2}^{\frac{d+1}{2}} \frac{(-1)^{p-2} p !(d-3) ! \alpha_{p}}{2^{2} 2 !(p-2) !(d+1-2 p) ! \ell_{\mathrm{eff}}^{2(p-2)}} \\
= & \left(\sum_{p=2}^{\frac{d-1}{2}} \frac{(-1)^{p-2} p !(d-3) ! \alpha_{p}}{2^{2} 2 !(p-2) !(d+1-2 p) ! \ell_{\mathrm{eff}}^{2(p-2)}}\right. \\
& \left.+\frac{(-1)^{\left(\frac{d+1}{2}\right)-2}\left(\frac{d+1}{2}\right) !(d-3) !}{2^{2} 2 !\left(\left(\frac{d+1}{2}\right)-2\right) ! \ell_{\mathrm{eff}}^{2\left(\left(\frac{d+1}{2}\right)-2\right)}}\left(\frac{2}{d+1} \sum_{p=1}^{\frac{d-1}{2}} \frac{p \alpha_{p}(-1)^{\frac{d+3}{2}-p}}{(d+1-2 p) !}\left(\ell_{\mathrm{eff}}\right)^{(d+1-2 p))}\right)\right) \\
= & \sum_{p=2}^{\frac{d-1}{2}}\left(\frac{(-1)^{p}(d-3) ! p \alpha_{p}}{2^{2} 2 !(d+1-2 p) ! \ell_{\mathrm{eff}}^{2(p-2)}}\left((p-1)-\left(\frac{d-1}{2}\right)\right)\right)+\frac{\alpha_{1}}{2^{4}(d-2)}\left(\ell_{\mathrm{eff}}\right)^{2} \\
= & \sum_{p=1}^{\frac{d-1}{2}}\left(\frac{(-1)^{p+1}(d-3) ! p \alpha_{p}}{2^{4}(d-2 p) ! \ell_{\mathrm{eff}}^{2(p-2)}}\right) \\
= & \frac{\ell_{\mathrm{eff}}^{2}}{2^{4}(d-2)} \Delta^{(1)}\left(\ell_{\mathrm{eff}}^{-2}\right) .
\end{aligned}
$$

Therefore, $\mathcal{F}^{2}$ term is proportional to the first degeneracy condition. Consequently -for degenerate points- the Noether prepotential is not linear but of higher order in $\mathcal{F}$, such that it is of normalizable order (in agreement with the results of ref. [45].

Now, we consider the coefficient of the $\mathcal{F}^{3}$ term, which is given by

$$
\begin{aligned}
c_{3}= & \sum_{p=3}^{\frac{d+1}{2}} \frac{(-1)^{p-3}(d-5) ! p ! a_{p}}{2^{3} 3 !(p-3) !(d+1-2 p) ! \ell_{\mathrm{eff}}^{2(p-3)}} \\
= & \left(\sum_{p=3}^{\frac{d-1}{2}} \frac{(-1)^{p-3}(d-5) ! p ! a_{p}}{2^{3} 3 !(p-3) !(d+1-2 p) ! \ell_{\mathrm{eff}}^{2(p-3)}}\right. \\
& \left.+\frac{(-1)^{\frac{d-5}{2}}(d-5) !\left(\begin{array}{c}
d+1 \\
2
\end{array}\right) !}{2^{3} 3 !\left(\begin{array}{c}
d-5 \\
2
\end{array}\right) ! \ell_{\mathrm{eff}}^{d-5}} \frac{2}{d+1} \sum_{p=1}^{\frac{d-1}{2}} \frac{p a_{p}(-1)^{\frac{d+3}{2}-p}}{(d+1-2 p) !} \ell_{\mathrm{eff}}^{d+1-2 p}\right) \\
= & \left(\sum_{p=3}^{\frac{d-1}{2}} \frac{(-1)^{p}(d-5) ! p ! a_{p}}{2^{3} 3 !(p-3) !(d+1-2 p) ! \ell_{\mathrm{eff}}^{2(p-3)}} \frac{(d+1-2 p)(d+2 p-5)}{4(p-1)(p-2)}\right. \\
& \left.+\frac{(d-5) !(d-1)(d-3)}{2^{3} 3 ! 4 \ell_{\mathrm{eff}}^{d-5}}\left(-\frac{a_{1}}{(d-1) !} \ell_{\mathrm{eff}}^{d-1}+\frac{2 a_{2}}{(d-3) !} \ell_{\mathrm{eff}}^{d-3}\right)\right) \\
= & \sum_{p=1}^{\frac{d-1}{2}} \frac{(-1)^{p}(d-5) ! p !(d-3) a_{p}}{2^{5} 3 !(p-1) !(d-2 p) ! \ell_{\mathrm{eff}}^{2(p-2)}} \ell_{\mathrm{eff}}^{2}+\sum_{p=2}^{\frac{d-1}{2}} \frac{(-1)^{p}(d-5) ! p ! a_{p}}{2^{4} 3 !(p-2) !(d-2 p) ! \ell_{\mathrm{eff}}^{2(p-2)}} \ell_{\mathrm{eff}}^{2} \\
= & -\frac{\ell_{\mathrm{eff}}^{4}}{2^{5} 3 !(d-2)(d-4)}\left(\Delta^{(1)}+\frac{4}{(d-3) \ell_{\mathrm{eff}}^{2}} \Delta^{(2)}\right)
\end{aligned}
$$


which we see is proportional to a linear combination of the first and second degeneracy conditions. Then, we consider the coefficient of the $\mathcal{F}^{4}$ term, which is given by

$$
\begin{aligned}
c_{4}= & \sum_{p=4}^{\frac{d+1}{2}} \frac{(-1)^{p-4} p !(d-7) ! \alpha_{p}}{2^{4} 4 !(p-4) !(d+1-2 p) ! \ell_{\mathrm{eff}}^{2(p-4)}} \\
= & \sum_{p=4}^{\frac{d-1}{2}} \frac{(-1)^{p-4} p !(d-7) ! \alpha_{p}}{2^{4} 4 !(p-4) !(d+1-2 p) ! \ell_{\mathrm{eff}}^{2(p-4)}}+\frac{(-1)^{\frac{d+1}{2}-4}\left(\begin{array}{c}
d+1 \\
2
\end{array}\right) !(d-7) !}{2^{4} 4 !\left(\frac{d+1}{2}-4\right) ! \ell_{\mathrm{eff}}^{d-7}} \frac{2}{d+1} \sum_{p=1}^{\frac{d-1}{2}} \frac{(-1)^{\frac{d+3}{2}-p} p \alpha_{p}}{(d+1-2 p) !} \ell_{\mathrm{eff}}^{d+1-2 p} \\
= & \sum_{p=4}^{\frac{d-1}{2}} \frac{(-1)^{p-4} p !(d-7) ! \alpha_{p}}{2^{4} 4 !(p-4) !(d+1-2 p) ! \ell_{\mathrm{eff}}^{2(p-4)}}\left[1-\frac{(d-1)(d-3)(d-5)}{2^{3}(p-1)(p-2)(p-3)}\right] \\
& -\frac{(d-1)(d-3)(d-5)}{2^{3}} \sum_{p=1}^{3} \frac{(-1)^{p-4} p !(d-7) ! \alpha_{p}}{2^{4} 4 !(p-1) !(d+1-2 p) ! \ell_{\mathrm{eff}}^{2(p-4)}} .
\end{aligned}
$$

The square bracket expression in the last equality can be written as

$$
1-\frac{(d-1)(d-3)(d-5)}{2^{3}(p-1)(p-2)(p-3)}=-\frac{(d-2 p+1)\left(d^{2}-10 d+33+4 p^{2}+2 d p-22 p\right)}{2^{3}(p-1)(p-2)(p-3)},
$$

which upon substituted into the previous expression, one gets

$$
\begin{aligned}
c_{4}= & \frac{1}{2^{7} 4 !(d-2)(d-4)(d-6)} \\
& \times\left[\sum_{p=4}^{\frac{d-1}{2}} \frac{(-1)^{p+1} p !(d-2) ! \alpha_{p}}{(p-4) !(d-2 p) ! \ell_{\mathrm{eff}}^{2(p-4)}} \frac{d^{2}-10 d+33+4 p^{2}+2 d p-22 p}{(p-1)(p-2)(p-3)(d-3)(d-5)}\right. \\
& \left.+\sum_{p=1}^{3} \frac{(-1)^{p+1} p !(d-2) ! \alpha_{p}}{(p-1) !(d-2 p) ! \ell_{\mathrm{eff}}^{2(p-4)}} \frac{d-1}{d+1-2 p}\right] \\
= & \frac{1}{2^{7} 4 !(d-2)(d-4)(d-6)} \\
& \times\left[\ell_{\mathrm{eff}}^{6} \sum_{p=1}^{\frac{d-1}{2}} \frac{(-1)^{p+1} p !(d-2) ! \alpha_{p}}{(p-1) !(d-2 p) ! \ell_{\mathrm{eff}}^{2(p-1)}}+\frac{4 \ell_{\mathrm{eff}}^{4}}{d-3} \sum_{p=2}^{\frac{d-1}{2}} \frac{(-1)^{p+1} p !(d-2) ! \alpha_{p}}{2 !(p-2) !(d-2 p) ! \ell_{\mathrm{eff}}^{2(p-2)}}\right. \\
& \left.+\frac{4 ! \ell_{\mathrm{eff}}^{2}}{(d-3)(d-5)} \sum_{p=3}^{\frac{d-1}{2}} \frac{(-1)^{p+1} p !(d-2) ! \alpha_{p}}{3 !(p-3) !(d-2 p) ! \ell_{\mathrm{eff}}^{2(p-3)}}\right] \\
= & \frac{\ell_{\mathrm{eff}}^{6}}{2^{7} 4 !(d-2)(d-4)(d-6)}\left[\Delta^{(1)}+\frac{4}{(d-3) \ell_{\mathrm{eff}}^{2}} \Delta^{(2)}+\frac{24}{(d-3)(d-5) \ell_{\mathrm{eff}}^{4}} \Delta^{(3)}\right] .
\end{aligned}
$$

We infer from the previous coefficients that the generic term is given by

$$
c_{i \geq 3}=\frac{(-1)^{i} \ell_{\mathrm{eff}}^{2 i-2}}{2^{2 i-1} i ! \prod_{j=1}^{i-1}(d-2 j)}\left(\Delta^{(1)}+\sum_{l=2}^{i-1} \frac{2^{l-1} l !}{\left(\prod_{m=1}^{l-1}(d-1-2 m)\right) \ell_{\mathrm{eff}}^{2 l-2}} \Delta^{(l)}\right)
$$


which explicitly reproduces the coefficients up to and including $c_{4}$. Using Mathematica, for arbitrary $c_{i}$ we have verified (C.6) for the particular Lovelock Unique Vacuum theories considered in ref. [54]. Finally, since

$$
c_{i}=\sum_{l=1}^{i-1} p_{(i, l)} \Delta^{(l)},
$$

we have

$$
\begin{aligned}
p_{(2,1)} & =\frac{\ell_{\mathrm{eff}}^{2}}{2^{3} 2 !(d-2)} \quad p_{(i \geq 3, l=1)}=\frac{(-1)^{i} \ell_{\mathrm{eff}}^{2 i-2}}{2^{2 i-1} i ! \prod_{j=1}^{i-1}(d-2 j)} \\
p_{(i \geq 3, l \geq 2)} & =\frac{(-1)^{i} \ell_{\mathrm{eff}}^{2(i-l)} 2^{l-2 i} l !}{i !\left(\prod_{j=1}^{i-1}(d-2 j)\right)\left(\prod_{m=1}^{l-1}(d-1-2 m)\right)} .
\end{aligned}
$$

\section{Noether prepotential from $P(\mathcal{F})$}

The Noether prepotential can be obtained by computing the on-shell variation of the bulk gravity action. This prepotential is important for computing the asymptotic charges of the theory $[40,45]$, as it is a covariant charge density that has to be integrated over codimension 2 surfaces at infinity.

Starting from the bulk $P(\mathcal{F})$ defined in eq. (3.15)

$$
P_{(d+1),\left\{\alpha_{p}\right\}}(\mathcal{F})=\sum_{j=2}^{\frac{d+1}{2}} c_{j} \delta_{\mu_{1} \cdots \mu_{2 j}}^{\nu_{1} \cdots \nu_{2 j}} \mathcal{F}_{\nu_{1} \nu_{2}}^{\mu_{1} \mu_{2}} \cdots \mathcal{F}_{\nu_{2 j-1} \nu_{2 j}}^{\mu_{2 j-1} \mu_{2 j}},
$$

where $c_{j}$ is given in eq. (3.16), we have

$$
I_{L}=\frac{1}{16 \pi G} \int_{M} d^{d+1} x \sqrt{-\mathcal{G}} P_{(d+1),\left\{\alpha_{p}\right\}}(\mathcal{F})+\tau_{d} \chi(M),
$$

for the renormalized Lovelock-AdS action (for odd $d$ ). Its on-shell variation is

$$
\begin{aligned}
\delta I_{L}= & \frac{1}{16 \pi G} \int_{\partial M} d^{d} x \sqrt{-h} \sum_{j=2}^{\frac{d+1}{2}}\left(-2 j c_{j}\right) \delta_{i_{1} \cdots i_{2 j-1}}^{k_{1} \cdots k_{2 j-1}} \mathcal{F}_{k_{1} k_{2}}^{i_{1} i_{2}} \cdots \mathcal{F}_{k_{2 j-3} k_{2 j-2}}^{i_{2 j-3} i_{2 j-2}} \\
& \times\left(\left(h^{-1} \delta h\right)_{l}^{i_{2 j-1}} K_{k_{2 j-1}}^{l}+2 \delta K_{k_{2 j-1}}^{i_{2 j-1}}\right)
\end{aligned}
$$

neglecting boundary terms at the AdS boundary. Therefore, in the case of non-degenerate theories, assuming asymptotic conformal flatness [44] and considering that $\mathcal{F}$ has the fall-off of the normalizable mode, we have that at the normalizable order

$$
\begin{aligned}
\delta I_{L} & =\frac{1}{16 \pi G} \int_{\partial M} d^{d} x \sqrt{-h}\left(-4 c_{2}\right) \delta_{j_{1} j_{2} j_{3}}^{i_{1} i_{2} i_{3}} \mathcal{F}_{i_{1} i_{2}}^{j_{1} j_{2}}\left(h^{-1} \delta h\right)_{l}^{j_{3}} K_{i_{3}}^{l}, \\
& =\frac{1}{16 \pi G} \int_{\partial M} d^{d} x \sqrt{-h}\left(\frac{-16 c_{2}}{\ell_{\mathrm{eff}}}\right) \mathcal{E}_{j}^{i}\left(h^{-1} \delta h\right)_{i}^{j},
\end{aligned}
$$


where we have taken just the leading order in the FG expansion of the extrinsic curvature $K_{j_{3}}^{l}=\delta_{j_{3}}^{l} / \ell_{\text {eff }}$, the electric part of the Weyl tensor $\mathcal{E}_{j}^{i}=\left(-W_{j l}^{i l}\right)$ and the fact that $W \simeq \mathcal{F}$ up to the normalizable order.

Finally, noting from eq. (C.1) that

$$
c_{2}=\sum_{p=2}^{\frac{d+1}{2}} \frac{(-1)^{p} p !(d-3) ! \alpha_{p}}{8(p-2) !(d+1-2 p) ! \ell_{\mathrm{eff}}^{2(p-2)}}=\frac{\ell_{\mathrm{eff}}^{2}}{2^{4}(d-2)} \Delta^{(1)}\left(\ell_{\mathrm{eff}}^{-2}\right)
$$

with $\Delta^{(1)}$ defined as in eq. (2.9), we have

$$
\delta I_{L}=-\frac{\ell_{\mathrm{eff}}}{16 \pi G(d-2)} \Delta^{(1)}\left(\ell_{\mathrm{eff}}^{-2}\right) \int_{\partial M} d^{d} x \sqrt{-h} \mathcal{E}_{j}^{i}\left(h^{-1} \delta h\right)_{i}^{j},
$$

from which we can directly read the Noether prepotential $\tau_{j}^{i}$

$$
\delta I_{L}=\int_{\partial M} d^{d} x \sqrt{-h}\left(\frac{1}{2} \tau_{j}^{i}\right)\left(h^{-1} \delta h\right)_{i}^{j},
$$

such that

$$
\tau_{i}^{j}=-\frac{\ell_{\mathrm{eff}}}{8 \pi G(d-2)} \Delta^{(1)}\left(\ell_{\mathrm{eff}}^{-2}\right) \mathcal{E}_{j}^{i},
$$

in agreement with ref. [45]. As $\Delta^{(1)}\left(\ell_{\text {eff }}^{-2}\right)=1$ for Einstein-AdS, this reproduces the known result for the Noether prepotential in this case.

\section{D.1 Degenerate case}

In the first-degenerate case, we have that $\Delta^{(1)}\left(\ell_{\text {eff }}^{-2}\right)=0$, implying $c_{2}=0$, and $\Delta^{(k>1)}\left(\ell_{\text {eff }}^{-2}\right) \neq 0$. From eq. (C.2)

$$
\begin{aligned}
c_{3} & =-\frac{\ell_{\mathrm{eff}}^{2}}{(d-2)(d-4) 2^{4} 3}\left(\frac{1}{(d-3)} \Delta^{(2)}+\frac{\ell_{\mathrm{eff}}^{2}}{4} \Delta^{(1)}\right) \\
& =-\frac{\ell_{\mathrm{eff}}^{2}}{(d-2)(d-3)(d-4) 2^{4} 3} \Delta^{(2)}\left(\ell_{\mathrm{eff}}^{-2}\right)
\end{aligned}
$$

and so the eq. (D.3) can be written to the lowest order as

$$
\delta I_{L}=\left(\frac{6 \ell_{\mathrm{eff}} \Delta^{(2)}\left(\ell_{\mathrm{eff}}^{-2}\right)}{16 \pi G(d-2)(d-3)(d-4) 2^{4} 3}\right) \int_{\partial M} d^{d} x \sqrt{-h} \delta_{j_{1} \cdots j_{5}}^{i_{1} \cdots i_{5}} \mathcal{F}_{i_{1} i_{2}}^{j_{1} j_{2}} \mathcal{F}_{i_{3} i_{4}}^{j_{3} j_{4}}\left(h^{-1} \delta h\right)_{i_{5}}^{j_{5}}
$$

Here, it is clear that the Noether prepotential is proportional to an antisymmetric contraction along boundary indices of $\mathcal{F}^{2}$. This is in agreement with ref. [45], where the asymptotic fall-off of said contraction was shown to be precisely the normalizable mode, making the Noether prepotential finite. 
In the $k$-degenerate case, we have $\Delta^{(q \leq k)}=0, \Delta^{(q>k)} \neq 0$, and because $c_{q}=$ $\sum_{i=1}^{q-1} p_{(q, i)} \Delta^{(i)}$ for some $p_{(q, i)}$ coefficients (as shown in eq. (C.8)), we have that $c_{q \leq k+1}=0$ and $c_{k+2}=p_{(k+2, k+1)} \Delta^{(k+1)}$. Thus, to lowest order we get

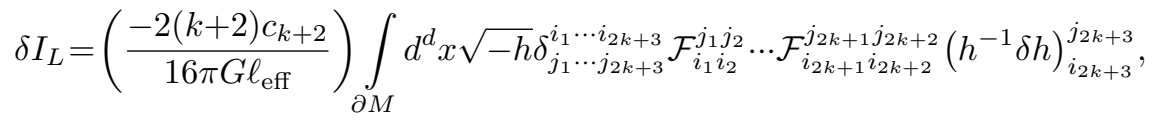

$$
\begin{aligned}
& =\left(\frac{-2(k+2) p_{(k+2, k+1)} \Delta^{(k+1)}\left(\ell_{\mathrm{eff}}^{-2}\right)}{16 \pi G \ell_{\mathrm{eff}}}\right) \int_{\partial M} d^{d} x \sqrt{-h} \delta_{j_{1} \cdots j_{2 k+3}}^{i_{1} \cdots i_{2 k+3}} \mathcal{F}_{i_{1} i_{2}}^{j_{1} j_{2}} \ldots \mathcal{F}_{i_{2 k+1} i_{2 k+2}}^{j_{2 k+1} j_{2 k+2}}\left(h^{-1} \delta h\right)_{i_{2 k+3}}^{j_{2 k+3}} .
\end{aligned}
$$

Note that the Noether prepotential is proportional to an antisymmetric contraction along boundary indices of $\mathcal{F}^{k+1}$, in agreement with ref. [45]. There it is shown that the eq. (D.11) falls-off asymptotically as the normalizable mode, thus rendering it finite. Direct comparison with ref. [45] fixes the $p_{(k+2, k+1)}$ coefficient to be that in eq. (3.23), such that for a $k$-degenerate theory, the lowest order coefficient in the bulk $P(\mathcal{F})$ is given by

$$
c_{k+2}=p_{(k+2, k+1)} \Delta^{(k+1)}\left(\ell_{\mathrm{eff}}^{-2}\right) \text {. }
$$

\section{E JM extremal surface for ball-shaped entangling region}

We proceed to verify that the spherical hemisphere is indeed the extremal surface of the JM functional for the case of ball-shaped entangling regions in pure AdS (dual to the ground state of a CFT in Minkowski spacetime). In order to see this, we consider that the bulk metric of the Poincaré patch of pure AdS (for a particular vacuum characterized by $\ell_{\text {eff }}^{2}$ ), is written in the FG gauge as

$$
\begin{gathered}
d s_{G}^{2}=G_{\mu \nu} d x^{\mu} d x^{\nu}=\frac{\ell_{\mathrm{eff}}^{2} d \rho^{2}}{4 \rho^{2}}+\frac{-d t^{2}+d r^{2}+r^{2} d \Omega_{d-2}^{2}}{\rho^{2}} \\
d \Omega_{d-2}^{2}=d \theta_{1}^{2}+\sin ^{2} \theta_{1} d \theta_{1}^{2}+\cdots+\sin ^{2} \theta_{1} \cdots \sin ^{2} \theta_{d-3}^{2} d \theta_{d-2}^{2} .
\end{gathered}
$$

Consider the spherical hemisphere

$$
\Sigma:\left\{t=\text { const. } ; r=\sqrt{R^{2}-\ell_{\mathrm{eff}}^{2} \rho}\right\}
$$

with $\left\{y^{\alpha}\right\}=\left\{\rho, \theta_{1}, \ldots, \theta_{d-2}\right\}$ being the worldvolume coordinates of $\Sigma$. The intrinsic metric

$$
\gamma_{\alpha \beta}=\frac{\partial x^{\mu}}{\partial y^{\alpha}} \frac{\partial x^{\nu}}{\partial y^{\beta}} G_{\mu \nu}
$$

on $\Sigma$ is then

$$
d s_{\gamma}^{2}=\gamma_{\alpha \beta} d y^{\alpha} d y^{\beta}=\frac{\ell_{\mathrm{eff}}^{2}}{4 \rho^{2}}\left(1+\frac{\ell_{\mathrm{eff}}^{2} \rho}{R^{2}-\ell_{\mathrm{eff}}^{2} \rho}\right) d \rho^{2}+\frac{\left(R^{2}-\ell_{\mathrm{eff}}^{2} \rho\right)}{\rho} d \Omega_{d-2}^{2} .
$$

In order to check that the ansatz (E.2) for the surface $\Sigma$ indeed minimizes the JM functional, we evaluate the corresponding equations of motion (4.7). Since $\Sigma$ is a constant curvature surface, we have

$$
\widehat{\mathcal{R}}_{\beta_{1} \beta_{2}}^{\alpha_{1} \alpha_{2}}[\gamma]=-\frac{1}{\ell_{\mathrm{eff}}^{2}} \delta_{\beta_{1} \beta_{2}}^{\alpha_{1} \alpha_{2}}
$$


for its Riemann curvature tensor. From eq. (4.7) we have

$$
\begin{aligned}
E_{J M} & =\sum_{p=0}^{\left\lfloor\frac{d-2}{2}\right\rfloor} \frac{(-1)^{p} \alpha_{(p+1)}(p+1)^{p}}{2^{p} \ell_{\mathrm{eff}}^{2 p}} \delta_{\beta_{1} \cdots \beta_{2 p+1}}^{\gamma_{1} \cdots \gamma_{2 p+1}} \mathcal{K}_{\gamma_{1}}^{\beta_{1}} \delta_{\gamma_{2} \gamma_{3}}^{\beta_{2} \beta_{3}} \cdots \delta_{\gamma_{2 p} \gamma_{2 p+1}}^{\beta_{2 p} \beta_{2 p+1}} \\
& =\sum_{p=0}^{\left\lfloor\frac{d-2}{2}\right\rfloor} \frac{(-1)^{p} \alpha_{(p+1)}(p+1)^{p}}{\ell_{\mathrm{eff}}^{2 p}} \delta_{\beta_{1} \cdots \beta_{2 p+1}}^{\gamma_{1} \cdots \gamma_{2 p+1}} \delta_{\gamma_{1}}^{\beta_{1}} \cdots \delta_{\gamma_{2 p}}^{\beta_{2 p}} \mathcal{K}_{\gamma_{2 p+1}}^{\beta_{2 p+1}} \\
& =\left(\sum_{p=0}^{\left\lfloor\frac{d-2}{2}\right\rfloor} \frac{(-1)^{p}(d-2) !(p+1) \alpha_{(p+1)}}{(d-2-2 p) ! \ell_{\mathrm{eff}}^{2 p}}\right) \operatorname{tr}[\mathcal{K}],
\end{aligned}
$$

using eqs. (A.2) and (A.1) from appendix A. Now, as shown in refs. [62, 66], the spherical hemisphere trivially satisfies $\operatorname{tr}[\mathcal{K}]=0$ as it is a minimal surface in the mathematical sense that it minimizes the area functional. In turn, this is equivalent to the statement that the RT surface is the correct one for the computation of HEE in Einstein-AdS gravity). Thus, the spherical hemisphere also satisfies $E_{J M}[\Sigma]=0$, and therefore it is the correct surface that minimizes the JM functional for the case of a ball-shaped entangling region in the CFT.

\section{F Evaluation of the JM functional and co-dimension 2 Kounterterm}

Here we evaluate the JM functional (5.6) and the co-dimension 2 Kounterterm used in the cancelation of divergences for the HEE. From eq. (5.5), the JM functional can be written as the sum of three terms, such that

$$
4 G S_{J M} \stackrel{\text { def. }}{=} I_{J M}=I_{J M}^{(1)}+I_{J M}^{(2)}+I_{J M}^{(3)},
$$

that we now proceed to evaluate. In order to simplify these terms, we employ the radial decomposition of the Riemann tensor of $\gamma_{\alpha \beta}$ given in eq. (5.2), and the FG-like expansions (5.1) and (5.3).

We start simplifying $I_{J M}^{(1)}$, where we have

$$
\begin{aligned}
I_{J M}^{(1)}= & \int_{\Sigma} d^{d-1} y \sqrt{\gamma} \sum_{p=1}^{\left\lfloor\frac{d-2}{2}\right\rfloor} \frac{(p+1) p \alpha_{(p+1)}}{2^{p-2}} \delta_{b_{1} \ldots b_{2 p-1}}^{a_{1} \ldots a_{2 p-1}}\left(\frac{1}{N} \partial_{\rho} k_{a_{1}}^{b_{1}}-k_{c}^{b_{1}} k_{a_{1}}^{c}\right) \\
& \times\left(\mathcal{R}^{b_{2} b_{3}}{ }_{a_{2} a_{3}}-2 k_{a_{2}}^{b_{2}} k_{a_{3}}^{b_{3}}\right) \cdots\left(\mathcal{R}^{b_{2 p-2} b_{2 p-1}} a_{a_{2 p-2} a_{2 p-1}}-2 k_{a_{2 p-2}}^{b_{2 p-2}} k_{a_{2 p-1}}^{b_{2 p-1}}\right) \\
= & \int_{\Sigma} d^{d-1} y \sqrt{\gamma} \sum_{p=1}^{\left\lfloor\frac{d-2}{2}\right\rfloor} \sum_{m=0}^{p-1} \sum_{s=0}^{2(p-1-m)} \frac{(-1)^{p-m}(p+1) !(2 p-2 m-2) !(d-2 p+s) ! \alpha_{(p+1)}}{2^{m-1} m ! s !(p-1-m) !(2 p-2 m-2-s) !(d-1-2 p) ! \ell_{\mathrm{eff}}^{s+2}} \\
& \times \rho^{2 p-m-s-2} \delta_{b_{1} \cdots b_{2 p-s-2}}^{a_{1} \cdots a_{2 p-s-2}} \mathcal{R}_{a_{1} a_{2}}^{b_{1} b_{2}}[\sigma] \cdots \mathcal{R}_{a_{2 m-1} a_{2 m}}^{b_{2 m-1} b_{2 m}}[\sigma]\left(k^{(2)}\right)_{a_{2 m+1}}^{b_{2 m+1}} \ldots \\
& \times\left(k^{(2)}\right)_{a_{2 p-s-2}}^{b_{2 p-s-2}}+\cdots,
\end{aligned}
$$

where the ellipsis indicates higher order terms and $\mathcal{R}^{a_{1} a_{2}}{ }_{b_{1} b_{2}}[\sigma]$ indicates the Riemann tensor of the $\sigma_{a b}$ metric. Finally, we isolate the leading and next-to-leading order divergences, 
to obtain

$$
\begin{aligned}
I_{J M}^{(1)}=\int_{\Sigma} d^{d-1} y \sqrt{\gamma} \sum_{p=1}^{\left\lfloor\frac{d-2}{2}\right\rfloor} \frac{(-1)^{p} 2(p+1) p \alpha_{(p+1)}}{(d-1-2 p) !}\left(\frac{(d-2) !}{\ell_{\mathrm{eff}}^{2 p}}+2(p-1) \rho \frac{(d-3) !}{\ell_{\mathrm{eff}}^{2 p-1}} \operatorname{tr}\left[k_{(2)}\right]\right. \\
\left.-(p-1) \rho \frac{(d-4) !}{\ell_{\mathrm{eff}}^{2 p-2}} \mathcal{R}[\sigma]\right)+\cdots .
\end{aligned}
$$

Considering the next term $I_{J M}^{(2)}$, we can write

$$
\begin{aligned}
I_{J M}^{(2)}= & \int_{\Sigma} d^{d-1} y \sqrt{\gamma} \sum_{p=2}^{\left\lfloor\frac{d-2}{2}\right\rfloor} \frac{(p+1)(p-1) p \alpha_{(p+1)}}{2^{p-2}} \delta_{b_{1} \ldots b_{2 p-1}}^{a_{1} \ldots a_{2 p-1}} \widehat{\mathcal{R}}_{a_{1} a_{2}}^{b_{1} \rho} \widehat{\mathcal{R}}_{a_{3} \rho}^{b_{2} b_{3}} \widehat{\mathcal{R}}_{a_{4} a_{5}}^{b_{4} b_{5}} \ldots \widehat{\mathcal{R}}_{a_{2 p-2} a_{2 p-1}}^{b_{2 p-2} b_{2 p-1}} \\
= & \int_{\Sigma} d^{d-1} y \sqrt{\gamma} \sum_{p=2}^{\left\lfloor\frac{d-2}{2}\right\rfloor} \frac{(p+1)(p-1) p \alpha_{(p+1)}}{2^{p-4}} \delta_{b_{1} \ldots b_{2 p-1}}^{a_{1} \ldots a_{2 p-1}} \nabla_{a_{1}} k_{a_{2}}^{b_{1}} \nabla^{b_{2}} k_{a_{3}}^{b_{3}}\left(\mathcal{R}_{a_{4} a_{5}}^{b_{4} b_{5}}-2 k_{a_{4}}^{b_{4}} k_{a_{5}}^{b_{5}}\right) \\
& \times \cdots\left(\mathcal{R}_{a_{2 p-2} b_{2 p-1}}^{b_{2 p-2} b_{2 p-1}}-2 k_{a_{2 p-2}}^{b_{2 p-2}} k_{a_{2 p-1}}^{b_{2 p-1}}\right) .
\end{aligned}
$$

From the expansion of the extrinsic curvature $k_{a b}$ in eq. (5.3), the term $\nabla_{a_{1}} k_{a_{2}}^{b_{1}}$ is $O(\rho)$, and $\nabla^{b_{2}} k_{a_{3}}^{b_{3}}$ is $O\left(\rho^{2}\right)$. Hence $\nabla_{a_{1}} k_{a_{2}}^{b_{1}} \nabla^{b_{2}} k_{a_{3}}^{b_{3}}$ is already $O\left(\rho^{3}\right)$, implying $I_{J M}^{(2)}$ does not contribute up to the next-to-leading divergence in $\rho$ and so can be neglected.

Finally, using eqs. (5.1)-(5.3) we can simplify $I_{J M}^{(3)}$ as

$$
\begin{aligned}
I_{J M}^{(3)}= & \int_{\Sigma} d^{d-1} y \sqrt{\gamma} \sum_{p=0}^{\left\lfloor\frac{d-2}{2}\right\rfloor} \frac{(p+1) \alpha_{(p+1)}}{2^{p}} \delta_{b_{1} \ldots b_{2 p}}^{a_{1} \ldots a_{2 p}}\left(\mathcal{R}_{a_{1} a_{2}}^{b_{1} b_{2}}-2 k_{a_{1}}^{b_{1}} k_{a_{2}}^{b_{2}}\right) \cdots\left(\mathcal{R}_{a_{2 p-1} a_{2 p}}^{b_{2 p-1} b_{2 p}}-2 k_{a_{2 p-1}}^{b_{2 p-1}} k_{a_{2 p}}^{b_{2 p}}\right) \\
= & \int_{\Sigma} d^{d-1} y \sqrt{\gamma} \sum_{m=0}^{\left\lfloor\frac{d-2}{2}\right\rfloor} \sum_{p=m}^{\left\lfloor\frac{d-2}{2}\right\rfloor} \sum_{s=0}^{2(p-m)}(-1)^{p-m} \frac{(p+1) \alpha_{(p+1)} p !(2 p-2 m) !(d-2-2 p+s) !}{2^{m} \ell_{\mathrm{eff}}^{s} m !(p-m !) s !(2 p-2 m-s) !(d-2-2 p) !} \\
& \times \rho^{2 p-m-s} \delta_{b_{1} \cdots b_{2 p-1}}^{a_{1} \cdots a_{2 p-s}} \mathcal{R}^{b_{1} b_{2}}{ }_{a_{1} a_{2}}[\sigma] \cdots \mathcal{R}^{b_{2 m-1} b_{2 m}}{ }_{a_{2 m-1} a_{2 m}}^{b_{2 m}}[\sigma]\left(k^{(2)}\right)_{a_{2 m+1}}^{b_{2 m+1}} \cdots\left(k^{(2)}\right)_{a_{2 p-s}}^{b_{2 p-s}}+\cdots
\end{aligned}
$$

Isolating the leading and next-to-leading orders in $\rho$ yields

$$
\begin{aligned}
I_{J M}^{(3)}=\int_{\Sigma} d^{d-1} y \sqrt{\gamma} \sum_{p=0}^{\left\lfloor\frac{d-2}{2}\right\rfloor}(-1)^{p} & \frac{(p+1) \alpha_{(p+1)}}{(d-2-2 p) !}\left(\frac{(d-2) !}{\ell_{\mathrm{eff}}^{2 p}}-\rho p\left(\frac{(d-4) !}{\ell_{\mathrm{eff}}^{2 p-2}} \mathcal{R}[\sigma]\right.\right. \\
& \left.\left.+2 \frac{(d-3) !}{\ell_{\mathrm{eff}}^{2 p}}\left(\operatorname{tr}\left[\sigma^{(2)}\right]+\frac{\ell_{\mathrm{eff}}^{2} \kappa_{a}^{(i) a} \kappa_{b}^{(i) b}}{2(d-2)}\right)\right)\right)+\cdots
\end{aligned}
$$

while implementing eq. (F.2) along with (F.5) results in eq. (5.6) for the JM functional $I_{J M}=I_{J M}^{(1)}+I_{J M}^{(3)}$ up to the next-to-leading divergent order. 
Now, we focus on the analysis of the Kounterterm $I_{K T}$. For odd $d$, we have

$$
\begin{aligned}
I_{K T}^{\text {odd }}= & 4 G S_{K T}^{\text {odd }}=\left\lfloor\frac{d+1}{2}\right\rfloor c_{d} \int_{\partial \Sigma} d^{d-2} y B_{d-2} \\
= & \left(\frac{d+1}{2}\right) c_{d}(-(d-1)) \int_{\partial \Sigma} d^{d-2} y \sqrt{\tilde{\gamma}} \int_{0}^{1} d t \delta_{b_{1} \cdots b_{d-2}}^{a_{1} \cdots a_{d-2}} k_{a_{1}}^{b_{1}}\left(\frac{1}{2} \mathcal{R}_{a_{2} a_{3}}^{b_{2} b_{3}}-t^{2} k_{a_{2}}^{b_{2}} k_{a_{3}}^{b_{3}}\right) \\
& \times \cdots\left(\frac{1}{2} \mathcal{R}_{a_{d-3} a_{d-2}}^{b_{d-3} b_{d-2}}-t^{2} k_{a_{d-3}}^{b_{d-3}} k_{a_{d-2}}^{b_{d-2}}\right)
\end{aligned}
$$

where the parametric integral $(P I)$ can be expanded as

$$
\begin{aligned}
P I= & \int_{0}^{1} d t \delta_{b_{1} \cdots b_{d-2}}^{a_{1} \cdots a_{d-2}} k_{a_{1}}^{b_{1}}\left(\frac{1}{2} \mathcal{R}_{a_{2} a_{3}}^{b_{2} b_{3}}-t^{2} k_{a_{2}}^{b_{2}} k_{a_{3}}^{b_{3}}\right) \cdots\left(\frac{1}{2} \mathcal{R}_{a_{d-3} b_{d-2}}^{b_{d-3} b_{d-2}}-t^{2} k_{a_{d-3}}^{b_{d-3}} k_{a_{d-2}}^{b_{d-2}}\right) \\
= & \sum_{m=0}^{\frac{d-3}{2}} \frac{\left(\frac{d-3}{2}\right) !(-1)^{\frac{d-3}{2}}-m}{m !\left(\frac{d-3}{2}-m\right) ! 2^{m}}\left(\int_{0}^{1} d t t^{d-3-2 m}\right) \delta_{b_{1} \cdots b_{d-2}}^{a_{1} \cdots a_{d-2}} \mathcal{R}_{a_{1} a_{2}}^{b_{1} b_{2}} \cdots \mathcal{R}_{a_{2 m-1} a_{2 m}}^{b_{2 m-1} b_{2 m}} k_{a_{2 m+1}}^{b_{2 m+1} \cdots k_{a_{d-2}}^{b_{d-2}}} \\
= & \sum_{m=0}^{\frac{d-3}{2}} \sum_{s=0}^{d-2-2 m} \frac{(-1)^{\frac{d-3}{2}-m}\left(\frac{d-3}{2}\right) !(d-3-2 m) ! \epsilon^{d-2-m-s}}{m !\left(\frac{d-3}{2}-m\right) !(d-2-2 m-s) ! 2^{m} \ell_{\mathrm{eff}}^{s}} \delta_{b_{1} \cdots b_{d-2-s}}^{a_{1} \cdots a_{d-2-s}} \\
& \times \mathcal{R}_{a_{1} a_{2}}^{b_{1} b_{2}}[\sigma] \cdots \mathcal{R}_{a_{2 m-1} b_{2 m}}^{b_{2 m-1} b_{2 m}}[\sigma]\left(k^{(2)}\right)_{a_{2 m+1}}^{b_{2 m+1}} \cdots\left(k^{(2)}\right)_{a_{d-2-s}}^{b_{d-2-s}}+\cdots
\end{aligned}
$$

using the FG-like expansions for the curvatures of $\partial \Sigma$ evaluated at the cutoff radius $\rho=\epsilon$. Up to the next-to-leading order, we then have

$$
P I=(-1)^{\frac{d-3}{2}}(d-3) !\left(\frac{1}{\ell_{\mathrm{eff}}^{d-2}}-\epsilon\left(\frac{\mathcal{R}^{(0)}}{2(d-4) \ell_{\mathrm{eff}}^{d-4}}+\frac{1}{\ell_{\mathrm{eff}}^{d-2}}\left(\operatorname{tr}\left[\sigma^{(2)}\right]+\frac{\ell_{\mathrm{eff}}^{2} \kappa_{a}^{(i) a} \kappa_{b}^{(i) b}}{2(d-2)}\right)\right)\right)+\cdots
$$

Noting that

$$
\sqrt{\widetilde{\gamma}}=\frac{\sqrt{\sigma^{(0)}}}{\epsilon^{\frac{d-2}{2}}}\left(1+\frac{\epsilon}{2} \operatorname{tr}\left[\sigma^{(2)}\right]+\mathcal{O}\left(\epsilon^{2}\right)\right)
$$

we then obtain

$$
\begin{aligned}
I_{K T}^{\mathrm{odd}}= & -\left(\frac{d+1}{2}\right)(d-1) c_{d} \int_{\partial \Sigma} d^{d-2} y \sqrt{\tilde{\gamma}} P I \\
= & -\int_{\partial \Sigma} d^{d-2} y \frac{\sqrt{\sigma^{(0)}}}{(d-2) \epsilon^{\frac{d-2}{2}}} \sum_{p=0}^{\frac{d-3}{2}} \frac{(-1)^{p}(p+1)(d-1) ! \alpha_{(p+1)}}{(d-1-2 p) ! \ell_{\mathrm{eff}}^{2 p-1}} \\
& \times\left(1-\epsilon\left[\frac{\ell_{\mathrm{eff}}^{2}}{2(d-4)} \mathcal{R}^{(0)}+\frac{1}{2} \operatorname{tr}\left(\sigma^{(2)}\right)+\frac{\ell_{\mathrm{eff}}^{2}}{2(d-2)} \kappa_{a}^{(i) a} \kappa_{b}^{(i) b}\right]\right)+\cdots
\end{aligned}
$$

for odd $d$. 
For even $d$, the expression for the Kounterterm is

$$
\begin{aligned}
I_{K T}^{\mathrm{even}}= & 4 G S_{K T}^{\mathrm{even}}=\left\lfloor\frac{d+1}{2}\right\rfloor c_{d} \int_{\partial \Sigma} d^{d-2} y B_{d-2} \\
= & \left(\frac{d}{2}\right) c_{d}(-(d-1)) \int_{\partial \Sigma} d^{d-2} y \sqrt{\tilde{\gamma}} \int_{0}^{1} d t \int_{0}^{t} d s \delta_{b_{1} \cdots b_{d-3}}^{a_{1} \cdots a_{d-3}} k_{a_{1}}^{b_{1}} \\
& \times\left(\frac{1}{2} \mathcal{R}^{b_{2} b_{3}}{ }_{a_{2} a_{3}}-t^{2} k_{a_{2}}^{b_{2}} k_{a_{3}}^{b_{3}}+\frac{s^{2}}{\ell_{\mathrm{eff}}^{2}} \delta_{a_{2}}^{b_{2}} \delta_{a_{3}}^{b_{3}}\right) \cdots\left(\frac{1}{2} \mathcal{R}^{b_{d-4} b_{d_{d-4}-3} b_{d-3}}-t^{2} k_{a_{d-4}}^{b_{d-4}} k_{a_{d-3}}^{b_{d-3}}+\frac{s^{2}}{\ell_{\mathrm{eff}}^{2}} \delta_{a_{d-4}}^{b_{d-4}} \delta_{a_{d-3}}^{b_{d-3}}\right)
\end{aligned}
$$

where for this case, the boundary term $\int d^{d-2} y B_{d-2}$ was evaluated in ref. [44], and the computational procedure is analogous to the odd $d$ case. Hence $I_{K T}^{\text {odd }}=I_{K T}^{\text {even }}$, and the Kounterterm $I_{K T}$ has the same form for both odd and even $d$ as given in eq. (5.9) of the main text.

Open Access. This article is distributed under the terms of the Creative Commons Attribution License (CC-BY 4.0), which permits any use, distribution and reproduction in any medium, provided the original author(s) and source are credited.

\section{References}

[1] S. Ryu and T. Takayanagi, Holographic derivation of entanglement entropy from AdS/CFT, Phys. Rev. Lett. 96 (2006) 181602 [hep-th/0603001] [INSPIRE].

[2] M.T. Grisaru, A.E.M. van de Ven and D. Zanon, Four loop $\beta$-function for the $N=1$ and $N=2$ supersymmetric nonlinear sigma model in two-dimensions, Phys. Lett. B 173 (1986) 423 [INSPIRE].

[3] D.J. Gross and E. Witten, Superstring modifications of Einstein's equations, Nucl. Phys. B 277 (1986) 1 [INSPIRE].

[4] S.S. Gubser, I.R. Klebanov and A.M. Polyakov, Gauge theory correlators from noncritical string theory, Phys. Lett. B 428 (1998) 105 [hep-th/9802109] [INSPIRE].

[5] A. Buchel, J. Escobedo, R.C. Myers, M.F. Paulos, A. Sinha and M. Smolkin, Holographic GB gravity in arbitrary dimensions, JHEP 03 (2010) 111 [arXiv:0911.4257] [INSPIRE].

[6] J. de Boer, M. Kulaxizi and A. Parnachev, Holographic Lovelock gravities and black holes, JHEP 06 (2010) 008 [arXiv:0912.1877] [INSPIRE].

[7] R.C. Myers, M.F. Paulos and A. Sinha, Holographic studies of quasi-topological gravity, JHEP 08 (2010) 035 [arXiv: 1004.2055] [INSPIRE].

[8] X.O. Camanho, J.D. Edelstein and J.M. Sánchez De Santos, Lovelock theory and the AdS/CFT correspondence, Gen. Rel. Grav. 46 (2014) 1637 [arXiv:1309.6483] [INSPIRE].

[9] M. Sinamuli and R.B. Mann, Higher order corrections to holographic black hole chemistry, Phys. Rev. D 96 (2017) 086008 [arXiv:1706. 04259] [InSPIRE].

[10] P. Bueno, P.A. Cano and A. Ruipérez, Holographic studies of Einsteinian cubic gravity, JHEP 03 (2018) 150 [arXiv: 1802.00018] [INSPIRE].

[11] Y. Kats and P. Petrov, Effect of curvature squared corrections in AdS on the viscosity of the dual gauge theory, JHEP 01 (2009) 044 [arXiv:0712.0743] [INSPIRE]. 
[12] M. Brigante, H. Liu, R.C. Myers, S. Shenker and S. Yaida, Viscosity bound violation in higher derivative gravity, Phys. Rev. D 77 (2008) 126006 [arXiv:0712.0805] [INSPIRE].

[13] X.O. Camanho and J.D. Edelstein, Causality constraints in AdS/CFT from conformal collider physics and Gauss-Bonnet gravity, JHEP 04 (2010) 007 [arXiv:0911.3160] [INSPIRE].

[14] X.O. Camanho, J.D. Edelstein and M.F. Paulos, Lovelock theories, holography and the fate of the viscosity bound, JHEP 05 (2011) 127 [arXiv: 1010.1682] [INSPIRE].

[15] R.C. Myers and A. Sinha, Holographic c-theorems in arbitrary dimensions, JHEP 01 (2011) 125 [arXiv: 1011.5819] [INSPIRE].

[16] M. Mezei, Entanglement entropy across a deformed sphere, Phys. Rev. D 91 (2015) 045038 [arXiv:1411.7011] [INSPIRE].

[17] P. Bueno, R.C. Myers and W. Witczak-Krempa, Universality of corner entanglement in conformal field theories, Phys. Rev. Lett. 115 (2015) 021602 [arXiv:1505. 04804] [INSPIRE].

[18] R.-X. Miao, A holographic proof of the universality of corner entanglement for CFTs, JHEP 10 (2015) 038 [arXiv:1507.06283] [INSPIRE].

[19] P. Bueno, P.A. Cano, R.A. Hennigar and R.B. Mann, Universality of squashed-sphere partition functions, Phys. Rev. Lett. 122 (2019) 071602 [arXiv: 1808.02052] [INSPIRE].

[20] P. Bueno, P.A. Cano, R.A. Hennigar, V.A. Penas and A. Ruipérez, Partition functions on slightly squashed spheres and flux parameters, JHEP 04 (2020) 123 [arXiv:2001.10020] [INSPIRE].

[21] C. Teitelboim and J. Zanelli, Dimensionally continued topological gravitation theory in Hamiltonian form, Class. Quant. Grav. 4 (1987) L125 [INSPIRE].

[22] R.C. Myers, Higher derivative gravity, surface terms and string theory, Phys. Rev. D 36 (1987) 392 [INSPIRE].

[23] S.C. Davis, Generalized Israel junction conditions for a Gauss-Bonnet brane world, Phys. Rev. D 67 (2003) 024030 [hep-th/0208205] [INSPIRE].

[24] D. Grumiller, R.B. Mann and R. McNees, Dirichlet boundary value problem for Chern-Simons modified gravity, Phys. Rev. D 78 (2008) 081502 [arXiv:0803.1485] [INSPIRE].

[25] J.D. Bekenstein, Black holes and entropy, Phys. Rev. D 7 (1973) 2333 [InSPIRE].

[26] S.W. Hawking, Particle creation by black holes, Commun. Math. Phys. 43 (1975) 199 [Erratum ibid. 46 (1976) 206] [INSPIRE].

[27] R.M. Wald, Black hole entropy is the Noether charge, Phys. Rev. D 48 (1993) R3427 [gr-qc/9307038] [INSPIRE].

[28] V. Iyer and R.M. Wald, Some properties of Noether charge and a proposal for dynamical black hole entropy, Phys. Rev. D 50 (1994) 846 [gr-qc/9403028] [InSPIRE].

[29] L.-Y. Hung, R.C. Myers and M. Smolkin, On holographic entanglement entropy and higher curvature gravity, JHEP 04 (2011) 025 [arXiv: 1101.5813] [INSPIRE].

[30] D.V. Fursaev, A. Patrushev and S.N. Solodukhin, Distributional geometry of squashed cones, Phys. Rev. D 88 (2013) 044054 [arXiv:1306.4000] [INSPIRE]. 
[31] X. Dong, Holographic entanglement entropy for general higher derivative gravity, JHEP 01 (2014) 044 [arXiv: 1310.5713] [INSPIRE].

[32] J. Camps, Generalized entropy and higher derivative gravity, JHEP 03 (2014) 070 [arXiv:1310.6659] [INSPIRE].

[33] A. Bhattacharyya, M. Sharma and A. Sinha, On generalized gravitational entropy, squashed cones and holography, JHEP 01 (2014) 021 [arXiv:1308.5748] [INSPIRE].

[34] A. Bhattacharyya, A. Kaviraj and A. Sinha, Entanglement entropy in higher derivative holography, JHEP 08 (2013) 012 [arXiv: 1305.6694] [INSPIRE].

[35] P. Bueno, J. Camps and A.V. López, Holographic entanglement entropy for perturbative higher-curvature gravities, JHEP 04 (2021) 145 [arXiv: 2012.14033] [INSPIRE].

[36] D. Lovelock, The Einstein tensor and its generalizations, J. Math. Phys. 12 (1971) 498 [INSPIRE].

[37] T. Jacobson and R.C. Myers, Black hole entropy and higher curvature interactions, Phys. Rev. Lett. 70 (1993) 3684 [hep-th/9305016] [INSPIRE].

[38] R. Olea, Mass, angular momentum and thermodynamics in four-dimensional Kerr-AdS black holes, JHEP 06 (2005) 023 [hep-th/0504233] [INSPIRE].

[39] R. Olea, Regularization of odd-dimensional AdS gravity: Kounterterms, JHEP 04 (2007) 073 [hep-th/0610230] [INSPIRE].

[40] G. Kofinas and R. Olea, Universal regularization prescription for Lovelock AdS gravity, JHEP 11 (2007) 069 [arXiv:0708.0782] [INSPIRE].

[41] G. Anastasiou, I.J. Araya and R. Olea, Renormalization of entanglement entropy from topological terms, Phys. Rev. D 97 (2018) 106011 [arXiv:1712.09099] [INSPIRE].

[42] G. Anastasiou, I.J. Araya and R. Olea, Topological terms, $A d S_{2 n}$ gravity and renormalized entanglement entropy of holographic CFTs, Phys. Rev. D 97 (2018) 106015 [arXiv: 1803.04990] [INSPIRE].

[43] G. Anastasiou, I.J. Araya, C. Arias and R. Olea, Einstein-AdS action, renormalized volume/area and holographic Rényi entropies, JHEP 08 (2018) 136 [arXiv:1806.10708] [INSPIRE].

[44] G. Anastasiou, I.J. Araya, A. Guijosa and R. Olea, Renormalized AdS gravity and holographic entanglement entropy of even-dimensional CFTs, JHEP 10 (2019) 221 [arXiv: 1908.11447] [INSPIRE].

[45] G. Arenas-Henriquez, R.B. Mann, O. Mišković and R. Olea, Mass in Lovelock unique vacuum gravity theories, Phys. Rev. D 100 (2019) 064038 [arXiv:1905.10840] [INSPIRE].

[46] G. Arenas-Henriquez, O. Mišković and R. Olea, Vacuum degeneracy and conformal mass in Lovelock AdS gravity, JHEP 11 (2017) 128 [arXiv:1710.08512] [INSPIRE].

[47] A. Lewkowycz and J. Maldacena, Generalized gravitational entropy, JHEP 08 (2013) 090 [arXiv: 1304.4926] [INSPIRE].

[48] T. Nishioka, Entanglement entropy: holography and renormalization group, Rev. Mod. Phys. 90 (2018) 035007 [arXiv: 1801.10352] [INSPIRE].

[49] H. Casini, M. Huerta and R.C. Myers, Towards a derivation of holographic entanglement entropy, JHEP 05 (2011) 036 [arXiv:1102.0440] [INSPIRE]. 
[50] S. de Haro, S.N. Solodukhin and K. Skenderis, Holographic reconstruction of space-time and renormalization in the AdS/CFT correspondence, Commun. Math. Phys. 217 (2001) 595 [hep-th/0002230] [INSPIRE].

[51] G. Anastasiou, O. Mišković, R. Olea and I. Papadimitriou, Counterterms, Kounterterms, and the variational problem in AdS gravity, JHEP 08 (2020) 061 [arXiv: 2003.06425] [INSPIRE].

[52] G. Kofinas and R. Olea, Universal Kounterterms in Lovelock AdS gravity, Fortsch. Phys. 56 (2008) 957 [arXiv:0806.1197] [INSPIRE].

[53] R.C. Myers and A. Sinha, Seeing a c-theorem with holography, Phys. Rev. D 82 (2010) 046006 [arXiv: 1006.1263 ] [INSPIRE].

[54] D. Kastor and R.B. Mann, On black strings and branes in Lovelock gravity, JHEP 04 (2006) 048 [hep-th/0603168] [INSPIRE].

[55] M.T. Anderson, $L^{2}$ curvature and volume renormalization of ahe metrics on 4-manifolds, Math. Res. Lett. 8 (2001) 171.

[56] C.R. Graham, Volume and area renormalizations for conformally compact Einstein metrics, Rend. Circ. Mat. Palermo S 63 (2000) 31 [math/9909042] [INSPIRE].

[57] A. Chang, J. Qing and P. Yang, On the renormalized volumes for conformally compact Einstein manifolds, math.DG/0512376 [INSPIRE].

[58] S. Alexakis and R. Mazzeo, Renormalized area and properly embedded minimal surfaces in hyperbolic 3-manifolds, Commun. Math. Phys. 297 (2010) 621 [INSPIRE].

[59] P. Albin, Renormalizing curvature integrals on Poincaré-Einstein manifolds, Adv. Math. 221 (2009) 140 [math.DG/0504161] [INSPIRE].

[60] J. Kastikainen, Conical defects and holography in topological AdS gravity, Class. Quant. Grav. 37 (2020) 195010 [arXiv: 2006.02803] [INSPIRE].

[61] J. de Boer, M. Kulaxizi and A. Parnachev, Holographic entanglement entropy in Lovelock gravities, JHEP 07 (2011) 109 [arXiv:1101.5781] [INSPIRE].

[62] A. Bhattacharyya and M. Sharma, On entanglement entropy functionals in higher derivative gravity theories, JHEP 10 (2014) 130 [arXiv:1405.3511] [INSPIRE].

[63] A. Schwimmer and S. Theisen, Entanglement entropy, trace anomalies and holography, Nucl. Phys. B 801 (2008) 1 [arXiv:0802.1017] [InSPIRE].

[64] L.-Y. Hung, R.C. Myers, M. Smolkin and A. Yale, Holographic calculations of Rényi entropy, JHEP 12 (2011) 047 [arXiv: 1110.1084] [INSPIRE].

[65] E. Poisson, A relativist's toolkit: the mathematics of black-hole mechanics, Cambridge University Press, Cambridge, U.K. (2009) [INSPIRE].

[66] G. Anastasiou, J. Moreno, R. Olea and D. Rivera-Betancour, Shape dependence of renormalized holographic entanglement entropy, JHEP 09 (2020) 173 [arXiv:2002.06111] [INSPIRE].

[67] G. Anastasiou, I.J. Araya, J. Moreno, R. Olea and D. Rivera-Betancour, Renormalized holographic entanglement entropy for quadratic curvature gravity, arXiv:2102.11242 [INSPIRE]. 